\title{
GAUSSIAN POLYTOPES: VARIANCES AND LIMIT THEOREMS
}

\author{
DANIEL HUG, ${ }^{* * *}$ Albert-Ludwigs-Universität Freiburg \\ MATTHIAS REITZNER, ${ }^{* * *}$ Technische Universität Wien
}

\begin{abstract}
The convex hull of $n$ independent random points in $\mathbb{R}^{d}$, chosen according to the normal distribution, is called a Gaussian polytope. Estimates for the variance of the number of $i$-faces and for the variance of the $i$ th intrinsic volume of a Gaussian polytope in $\mathbb{R}^{d}, d \in \mathbb{N}$, are established by means of the Efron-Stein jackknife inequality and a new formula of Blaschke-Petkantschin type. These estimates imply laws of large numbers for the number of $i$-faces and for the $i$ th intrinsic volume of a Gaussian polytope as $n \rightarrow \infty$.

Keywords: Random point; convex hull; $f$-vector; intrinsic volume; geometric probability; normal distribution; Gaussian sample; stochastic geometry; variance; law of large numbers; limit theorem
\end{abstract}

2000 Mathematics Subject Classification: Primary 52A22; 60D05

Secondary $60 \mathrm{C} 05 ; 62 \mathrm{H} 10$

\section{Introduction and statement of results}

Let $X_{1}, \ldots, X_{n}$ be a Gaussian sample in $\mathbb{R}^{d}, d \in \mathbb{N}$, i.e. independent random points chosen according to the $d$-dimensional standard normal distribution with mean 0 and covariance matrix $\frac{1}{2} I_{d}$. Denote by $P_{n}=\left[X_{1}, \ldots, X_{n}\right]$ the convex hull of these random points, and call $P_{n}$ a Gaussian polytope. We are interested in geometric functionals such as the volume, the intrinsic volumes, and the number of $i$-dimensional faces of Gaussian polytopes. Most of the previous investigations on this topic were concerned with expectations of such functionals. The starting point of this line of research is marked by a classic paper by Rényi and Sulanke [22], in which the asymptotic behaviour of the expected number of vertices, E $f_{0}\left(P_{n}\right)$, of $P_{n}-$ and, thus, also that of the expected number of edges, E $f_{1}\left(P_{n}\right)$, as $n$ tends to infinity - was determined in the plane. This result was generalized by Raynaud [19], who investigated the asymptotic behaviour of the mean number of facets, $\mathrm{E} f_{d-1}\left(P_{n}\right)$, in arbitrary dimension. Both results are only particular cases of the formula

$$
\mathrm{E} f_{i}\left(P_{n}\right)=\frac{2^{d}}{\sqrt{d}}\left(\begin{array}{c}
d \\
i+1
\end{array}\right) \beta_{i, d-1}(\pi \ln n)^{(d-1) / 2}(1+o(1)),
$$

where $i \in\{0, \ldots, d-1\}$ and $d \in \mathbb{N}$, as $n \rightarrow \infty$. This follows, in arbitrary dimension, from work of Affentranger and Schneider [2] and Baryshnikov and Vitale [3]. Here, $f_{i}\left(P_{n}\right)$ denotes the number of $i$-faces of $P_{n}$ and $\beta_{i, d-1}$ is the internal angle of a regular $(d-1)$-simplex at one

Received 1 November 2004; revision received 27 January 2005.

Supported in part by the European Network PHD, MCRN-511953.

* Postal address: Mathematisches Institut, Albert-Ludwigs-Universität, Eckerstr. 1, D-79104 Freiburg i. Br., Germany. Email address: daniel.hug@math.uni-freiburg.de

** Postal address: Institut für Diskrete Mathematik und Geometrie, Technische Universität Wien, Wiedner Hauptstrasse 8-10, A-1040 Vienna, Austria. Email address: mreitzne@mail.zserv.tuwien.ac.at 
of its $i$-dimensional faces. Recently, a more direct proof of (1.1) and some additional relations, which cannot be derived from [2] and [3], were given in [9]. However, it turned out to be difficult to extend these results to higher moments of $f_{i}\left(P_{n}\right)$ and, thus, to prove limit theorems. An exception is the particular case $i=0$, where Hueter [7], [8] stated a central limit theorem,

$$
\frac{f_{0}\left(P_{n}\right)-\mathrm{E} f_{0}\left(P_{n}\right)}{\sqrt{\operatorname{var} f_{0}\left(P_{n}\right)}} \stackrel{\mathrm{D}}{\rightarrow} \mathcal{N}(0,1)
$$

as $n$ tends to infinity; here ' $\stackrel{\mathrm{D}}{\rightarrow}$ ' denotes convergence in distribution and $\mathcal{N}(0,1)$ is the (onedimensional) normal distribution. The asymptotic behaviour of the variance was asserted to be of the form

$$
\operatorname{var} f_{0}\left(P_{n}\right)=\bar{c}_{d}(\ln n)^{(d-1) / 2}(1+o(1)),
$$

with a constant $\bar{c}_{d}$, as $n \rightarrow \infty$. Most probably, it is difficult to establish such a precise limit relation for all $f_{i}\left(P_{n}\right), i \in\{1, \ldots, d-1\}$. Our first result provides an upper bound for the order of the variance of $f_{i}\left(P_{n}\right)$, for all $i \in\{0, \ldots, d-1\}$, which is of order $(\ln n)^{(d-1) / 2}$.

Theorem 1.1. Let $f_{i}\left(P_{n}\right)$ be the number of $i$-dimensional faces of a d-dimensional Gaussian polytope $P_{n}, d \in \mathbb{N}$. Then there exists a positive constant $c_{d}$, depending only on the dimension, such that

$$
\operatorname{var} f_{i}\left(P_{n}\right) \leq c_{d}(\ln n)^{(d-1) / 2}
$$

for all $i \in\{0, \ldots, d-1\}$.

Combining Chebyshev's inequality and (1.3), for all $\varepsilon>0$ we obtain

$$
\begin{aligned}
\mathrm{P}\left(\left|f_{i}\left(P_{n}\right)-\mathrm{E} f_{i}\left(P_{n}\right)\right|(\ln n)^{-(d-1) / 2} \geq \varepsilon\right) & \leq \varepsilon^{-2}(\ln n)^{-(d-1)} \operatorname{var} f_{i}\left(P_{n}\right) \\
& \leq \varepsilon^{-2} c_{d}(\ln n)^{-(d-1) / 2}
\end{aligned}
$$

and, thus, the random variable $f_{i}\left(P_{n}\right)$ satisfies a (weak) law of large numbers for all $d \in \mathbb{N}$ (the case $d=1$ is trivial). In fact, the law of $f_{i}\left(P_{n}\right)(\ln n)^{-(d-1) / 2}$ converges in probability to the law concentrated at a constant.

Corollary 1.1. For $d \in \mathbb{N}$ and $i \in\{0, \ldots, d-1\}$, the number of $i$-dimensional faces, $f_{i}\left(P_{n}\right)$, of a Gaussian polytope $P_{n}$ in $\mathbb{R}^{d}$ satisfies

$$
f_{i}\left(P_{n}\right)(\ln n)^{-(d-1) / 2} \longrightarrow \frac{2^{d}}{\sqrt{d}}\left(\begin{array}{c}
d \\
i+1
\end{array}\right) \beta_{i, d-1} \pi^{(d-1) / 2}
$$

in probability as $n \rightarrow \infty$.

Massé [15] deduced a corresponding weak law of large numbers for $d=2$ and $i=0$ from Hueter's central limit theorem (1.2).

Our method of proof also works for the volume and, more generally, the intrinsic volumes. Denote by $V_{i}\left(P_{n}\right)$ the $i$ th intrinsic volume of the Gaussian polytope $P_{n}$; hence, for instance, $V_{d}\left(P_{n}\right)$ is the volume, $2 V_{d-1}\left(P_{n}\right)$ is the surface area, and $V_{1}\left(P_{n}\right)$ is a multiple of the mean width of $P_{n}$. The expected values of the $i$ th intrinsic volumes were investigated by Affentranger [1], who proved that

$$
\mathrm{E} V_{i}\left(P_{n}\right)=\left(\begin{array}{l}
d \\
i
\end{array}\right) \frac{\kappa_{d}}{\kappa_{d-i}}(\ln n)^{i / 2}(1+o(1))
$$


for $i \in\{1, \ldots, d\}$ as $n$ tends to infinity, where $\kappa_{j}$ denotes the volume of the $j$-dimensional unit ball. The case $d=1$, which was not covered in [1], can be checked directly. Relation (1.4) was expected to hold, since a result of Geffroy [5] implies that the Hausdorff distance between $P_{n}$ and the $d$-dimensional ball of radius $(\ln n)^{1 / 2}$, centred at the origin, converges almost surely to 0 . However, it seems that (1.4) cannot be deduced directly from Geffroy's result.

In the planar case, Hueter also stated central limit theorems for $V_{1}\left(P_{n}\right)$ and $V_{2}\left(P_{n}\right)$ :

$$
\frac{V_{1}\left(P_{n}\right)-\mathrm{E} V_{1}\left(P_{n}\right)}{\sqrt{\operatorname{var} V_{1}\left(P_{n}\right)}} \stackrel{\mathrm{D}}{\rightarrow} \mathcal{N}(0,1) \quad \text { and } \quad \frac{V_{2}\left(P_{n}\right)-\mathrm{E} V_{2}\left(P_{n}\right)}{\sqrt{\operatorname{var} V_{2}\left(P_{n}\right)}} \stackrel{\mathrm{D}}{\rightarrow} \mathcal{N}(0,1) .
$$

The variances suggested by Hueter are of the form var $V_{i}\left(P_{n}\right)=\frac{1}{2} \pi^{3 / 2}(\ln n)^{i}(1+o(1))$. That her result cannot be correct can be seen from the following: if the stated asymptotic behaviour of the variances were true, this would immediately imply that

$$
\begin{aligned}
& \mathrm{P}\left(V_{1}\left(P_{n}\right) \leq 0\right) \rightarrow \Phi\left(-(4 \pi)^{1 / 4}\right), \\
& \mathrm{P}\left(V_{2}\left(P_{n}\right) \leq 0\right) \rightarrow \Phi\left(-(4 \pi)^{1 / 4}\right) .
\end{aligned}
$$

However, the stated probabilities would then be positive for large $n$, which obviously cannot hold. In the next theorem, we give an upper bound for the variances, for all $i=1, \ldots, d$ and $d \in \mathbb{N}$.

Theorem 1.2. Let $V_{i}\left(P_{n}\right)$ be the ith intrinsic volume of a Gaussian polytope $P_{n}$ in $\mathbb{R}^{d}, d \in \mathbb{N}$. Then there exists a positive constant $c_{d}$, depending only on the dimension, such that

$$
\operatorname{var} V_{i}\left(P_{n}\right) \leq c_{d}(\ln n)^{(i-3) / 2}
$$

for all $i \in\{1, \ldots, d\}$.

Let $X_{1}, X_{2}, \ldots$ be a sequence of independent random points that are identically distributed according to the $d$-dimensional normal distribution, and let $P_{n}=\left[X_{1}, \ldots, X_{n}\right]$. For $d=1$, the quantity $V_{1}\left(P_{n}\right)$ is the sample range of $X_{1}, \ldots, X_{n}$. Although its distribution and moments can be expressed as multiple integrals (see [18, Chapter 8], [13, Chapter 14], and [14]), explicit values are not available, in general. The asymptotic behaviour of var $V_{1}\left(P_{n}\right)$ for $d=1$ is deduced in Chapter 14 (see Equation (14.100)) of [13], which yields (with the present normalization)

$$
\operatorname{var} V_{1}\left(P_{n}\right)=\frac{1}{12} \pi^{2}(\ln n)^{-1}(1+o(1)) \quad \text { as } n \rightarrow \infty .
$$

A different extension of the univariate sample range to higher dimensions is given by the largest interpoint distance of the given random points. Limiting distributions have been considered in [16] and, in a more general framework, in [6]; still another extension was discussed in [10].

From (1.4) and (1.5), we obtain an additive weak law of large numbers for $i \in\{1,2\}$, i.e.

$$
V_{i}\left(P_{n}\right)-\left(\begin{array}{l}
d \\
i
\end{array}\right) \frac{\kappa_{d}}{\kappa_{d-i}}(\ln n)^{i / 2} \rightarrow 0
$$

in probability as $n \rightarrow \infty$. In order to derive a (multiplicative) strong law of large numbers for $i \in\{1, \ldots, d\}$, we set $n_{k}=2^{k}$. From the upper bound for the variance and Chebyshev's inequality, we deduce that

$$
\mathrm{P}\left(\left|V_{i}\left(P_{n_{k}}\right)-\mathrm{E} V_{i}\left(P_{n_{k}}\right)\right|\left(\ln n_{k}\right)^{-i / 2} \geq \varepsilon\right) \leq \varepsilon^{-2} c_{d}\left(\ln n_{k}\right)^{-(i+3) / 2} .
$$


Since

$$
\sum_{k \geq 1}\left(\ln n_{k}\right)^{-(i+3) / 2}=(\ln 2)^{-(i+3) / 2} \sum_{k \geq 1} k^{-(i+3) / 2}<\infty,
$$

(1.4) and the Borel-Cantelli lemma imply that

$$
V_{i}\left(P_{n_{k}}\right)\left(\ln n_{k}\right)^{-i / 2} \rightarrow\left(\begin{array}{l}
d \\
i
\end{array}\right) \frac{\kappa_{d}}{\kappa_{d-i}}
$$

with probability 1 as $k$ tends to infinity. Moreover, since $n \mapsto V_{i}\left(P_{n}\right)$ is increasing,

$$
V_{i}\left(P_{n_{k-1}}\right)\left(\ln n_{k}\right)^{-i / 2} \leq V_{i}\left(P_{n}\right)(\ln n)^{-i / 2} \leq V_{i}\left(P_{n_{k}}\right)\left(\ln n_{k-1}\right)^{-i / 2}
$$

for $n_{k-1} \leq n \leq n_{k}$, where $\left(\ln n_{k+1}\right) /\left(\ln n_{k}\right) \rightarrow 1$ by definition. Thus, (1.6) implies a strong law of large numbers.

Corollary 1.2. Let $V_{i}\left(P_{n}\right)$ be the ith intrinsic volume of a Gaussian polytope $P_{n}$ in $\mathbb{R}^{d}, d \in \mathbb{N}$. Then, for $i \in\{1, \ldots, d\}$,

$$
V_{i}\left(P_{n}\right)(\ln n)^{-i / 2} \rightarrow\left(\begin{array}{l}
d \\
i
\end{array}\right) \frac{\kappa_{d}}{\kappa_{d-i}}
$$

with probability 1 as $n \rightarrow \infty$.

This law of large numbers can also be deduced from a result of Geffroy [5].

The estimates for the variances obtained in Theorems 1.1 and 1.2 are based on the solution of another problem, which is of independent interest. Consider the random polytope $P_{n}$ and choose another independent random point $X$ according to the normal distribution. The question in which we are interested is the following: if $X \notin P_{n}$, how many facets of $P_{n}$ can be seen from $X$ ? We will determine the asymptotic behaviour of the expectation of the corresponding random variable as $n \rightarrow \infty$, and we will provide upper and lower bounds for its second moment.

In the following, let $F_{n}(X)$ be the number of facets of $P_{n}$ that can be seen from $X$; more precisely, we count the number of those facets of $P_{n}$ whose relative interiors are contained in the interior of the convex hull of $P_{n}$ and $X$. Note that $F_{n}(X)=0$ if $X$ is contained in $P_{n}$.

Theorem 1.3. Let $X, X_{1}, \ldots, X_{n}$ be independent random points in $\mathbb{R}^{d}, d \in \mathbb{N}$, that are identically distributed according to the d-dimensional normal distribution. Let $P_{d}^{(d-1)}$ denote a Gaussian polytope in $\mathbb{R}^{d-1}$. Then

$$
\lim _{n \rightarrow \infty} \mathrm{E} F_{n}(X) n(\ln n)^{-(d-1) / 2}=2^{d-1} \kappa_{d} \Gamma(d+1) \mathrm{E} V_{d-1}\left(P_{d}^{(d-1)}\right) .
$$

Furthermore, there is a positive constant $c_{d}$, depending only on the dimension, such that

$$
c_{d}^{-1} n^{-1}(\ln n)^{(d-1) / 2} \leq \mathrm{E} F_{n}(X)^{2} \leq c_{d} n^{-1}(\ln n)^{(d-1) / 2} .
$$

For more information on random polytopes, we refer the reader to the recent survey article by Schneider [25].

\section{Projections of high-dimensional simplices}

We want to give two interpretations of our results. The first one uses the fact that any orthogonal projection of a Gaussian sample is itself a Gaussian sample. We therefore make our notation more precise by writing $P_{n}^{(d)}$ for a Gaussian polytope in $\mathbb{R}^{d}$ that is the convex hull of 
$n$ normally distributed random points in $\mathbb{R}^{d}$. Let $\Pi_{i}: \mathbb{R}^{d} \rightarrow \mathbb{R}^{i}$ be the projection onto the first $i$ components $(i<d)$. For an arbitrary $i$-dimensional subspace of $\mathbb{R}^{d}$, which we identify with $\mathbb{R}^{i}$, we then obtain

$$
\varphi\left(\Pi_{i} P_{n}^{(d)}\right) \stackrel{\mathrm{D}}{=} \varphi\left(P_{n}^{(i)}\right),
$$

where ' $\stackrel{=}{=}$ means equality in distribution and $\varphi$ is any (measurable) functional on the convex polytopes.

Now let $P_{n+1}^{(n)}$ be a Gaussian simplex in $\mathbb{R}^{n}$. As a consequence of Corollary 1.2 and (2.1), we obtain a law of large numbers for projections of high-dimensional random simplices: for a fixed integer $i \geq 1$,

$$
V_{i}\left(\Pi_{i} P_{n+1}^{(n)}\right)(\ln n)^{-i / 2} \rightarrow \kappa_{i}
$$

in probability as $n \rightarrow \infty$. Moreover, for a fixed integer $i \geq 1$, (1.4) implies that

$$
\mathrm{E} V_{i}\left(\Pi_{i} P_{n+1}^{(n)}\right)=\mathrm{E} V_{i}\left(P_{n+1}^{(i)}\right)=\kappa_{i}(\ln n)^{i / 2}(1+o(1))
$$

as $n \rightarrow \infty$. An estimate of the variance can be deduced from Theorem 1.2. Thus, for $i \geq 1$, we have

$$
\operatorname{var} V_{i}\left(\Pi_{i} P_{n+1}^{(n)}\right) \leq c_{i}(\ln n)^{(i-3) / 2} .
$$

Finally, Kubota's theorem (see [26, Equation (4.6)] and [24, Equation (5.3.27)]), Hölder's inequality, and Theorem 1.2 yield the following asymptotic result for the $i$ th intrinsic volume of a high-dimensional Gaussian simplex (see the proof of Theorem 1.2 in Section 7 for a similar argument).

Corollary 2.1. Let $V_{i}\left(P_{n+1}^{(n)}\right)$ be the ith intrinsic volume of a Gaussian simplex in $\mathbb{R}^{n}$. Then, for any fixed integer $i \geq 1$,

$$
V_{i}\left(P_{n+1}^{(n)}\right) c_{n, i}^{-1}(\ln n)^{-i / 2} \rightarrow \kappa_{i}
$$

in probability as $n \rightarrow \infty$, where $c_{n, i}=\left(\begin{array}{l}n \\ i\end{array}\right) \kappa_{n} /\left(\kappa_{i} \kappa_{n-i}\right)$.

Another method of generating $n+1$ random points in $\mathbb{R}^{d}$ goes back to a suggestion of Goodman and Pollack. Let $R$ denote a random rotation of $\mathbb{R}^{n}$, let $\Pi_{d}^{*}:=\Pi_{d} \circ R$ (recall that $\Pi_{d}$ denotes the projection onto $\mathbb{R}^{d}$ ), and let $T^{(n)}$ be a regular simplex in $\mathbb{R}^{n}$. Then $\Pi_{d}^{*}\left(T^{(n)}\right)$ is a random polytope in $\mathbb{R}^{d}$ in the Goodman-Pollack model. It was proved, in [3], that

$$
\varphi\left(\Pi_{d}^{*} T^{(n)}\right) \stackrel{\mathrm{D}}{=} \varphi\left(P_{n+1}^{(d)}\right)
$$

for any affine invariant (measurable) functional $\varphi$ on the convex polytopes. Thus, if $f_{i}$ denotes the number of $i$-faces, (1.1) is equivalent to

$$
\mathrm{E} f_{i}\left(\Pi_{d}^{*} T^{(n)}\right)=\frac{2^{d}}{\sqrt{d}}\left(\begin{array}{c}
d \\
i+1
\end{array}\right) \beta_{i, d-1}(\pi \ln n)^{(d-1) / 2}(1+o(1))
$$

as $n \rightarrow \infty$, which is what was actually proved in [2]. By (2.2), the bound for the variance in Theorem 1.1 and the law of large numbers in Corollary 1.1 now give bounds and a law of large numbers, respectively, for $\Pi_{d}^{*} T^{(n)}$, i.e.

$$
\operatorname{var} f_{i}\left(\Pi_{d}^{*} T^{(n)}\right) \leq c_{d}(\ln n)^{(d-1) / 2}
$$


and, for $d \in \mathbb{N}$,

$$
f_{i}\left(\Pi_{d}^{*} T^{(n)}\right)(\ln n)^{-(d-1) / 2} \longrightarrow \frac{2^{d}}{\sqrt{d}}\left(\begin{array}{c}
d \\
i+1
\end{array}\right) \beta_{i, d-1} \pi^{(d-1) / 2}
$$

in probability, as $n$ tends to infinity.

For further information on the Goodman-Pollack model and related work of Vershik and Sporyshev [27], we refer the reader to [2].

\section{A new formula of Blaschke-Petkantschin type}

We work in the $d$-dimensional Euclidean space $\mathbb{R}^{d}$ with scalar product $\langle\cdot, \cdot\rangle$ and induced norm $\|\cdot\|$. The $d$-dimensional Lebesgue measure in $\mathbb{R}^{d}$ will be denoted by $\lambda_{d}$. We write $\mathbb{S}^{d-1}$ for the Euclidean unit $(d-1)$-sphere and $\sigma$ for the spherical Lebesgue measure (the dimension will be clear from the context). Recall that the convex hull of the points $x_{1}, \ldots, x_{m} \in \mathbb{R}^{d}$ is denoted by $\left[x_{1}, \ldots, x_{m}\right]$. If $P \subset \mathbb{R}^{d}$ is a (convex) polytope then we write $\mathcal{F}_{k}(P)$ for the set of its $k$-dimensional faces and $f_{k}(P)$ for the number of these $k$-faces, where $k \in\{0, \ldots, d\}$. The $k$-dimensional Lebesgue measure in a $k$-dimensional plane $E \subset \mathbb{R}^{d}$ is denoted by $\lambda_{E}$. Subspaces are endowed with the induced scalar product and norm. Finally, we write $\Gamma(\cdot)$ for the gamma function, recalling that $\Gamma(n+1)=n$ ! for $n \in \mathbb{N}$.

An important tool in our investigations will be a new formula of Blaschke-Petkantschin type. The classical affine Blaschke-Petkantschin formula (see, e.g. [23, Section II.12.3] and [26, Section 6.1]) states that

$$
\begin{aligned}
\int_{\mathbb{R}^{d}} & \cdots \int_{\mathbb{R}^{d}} f\left(x_{1}, \ldots, x_{d}\right) \prod_{j=1}^{d} \mathrm{~d} \lambda_{d}\left(x_{j}\right) \\
& =\Gamma(d) \int_{\mathcal{H}_{d-1}^{d}} \int_{H} \cdots \int_{H} f\left(x_{1}, \ldots, x_{d}\right) \lambda_{H}\left(\left[x_{1}, \ldots, x_{d}\right]\right) \prod_{j=1}^{d} \mathrm{~d} \lambda_{H}\left(x_{j}\right) \mathrm{d} \bar{\mu}(H)
\end{aligned}
$$

for any nonnegative measurable function $f:\left(\mathbb{R}^{d}\right)^{d} \rightarrow \mathbb{R}$. Here, $\bar{\mu}$ is the motion-invariant Haar measure on the affine Grassmannian $\mathcal{H}_{d-1}^{d}$ of hyperplanes in $\mathbb{R}^{d}$, normalized such that the measure of all hyperplanes hitting the Euclidean unit ball is equal to $d \kappa_{d}$. Any hyperplane $H$ with $0 \notin H$ can be parametrized (uniquely) by one of its unit normal vectors $u \in \mathbb{S}^{d-1}$ and its distance $t \geq 0$ to the origin, such that $H=\left\{y \in \mathbb{R}^{d}:\langle y, u\rangle=t\right\}$. Then we have

$$
\bar{\mu}(\cdot)=\int_{\mathbb{S}^{d-1}} \int_{0}^{\infty} \mathbf{1}_{\left\{t u+u^{\perp} \in \cdot\right\}} \mathrm{d} t \mathrm{~d} \sigma(u),
$$

where $u^{\perp}$ denotes the $(d-1)$-dimensional subspace of $\mathbb{R}^{d}$ orthogonal to $u$.

The affine Blaschke-Petkantschin formula relates the $d$-dimensional volume elements $\mathrm{d} \lambda_{d}\left(x_{j}\right)$ of points $x_{1}, \ldots, x_{d}$ to the differential $\mathrm{d} \bar{\mu}(H)$ of a hyperplane $H$ and the $(d-1)$ dimensional volume elements $\mathrm{d} \lambda_{H}\left(x_{j}\right)$ of points $x_{j} \in H, j=1, \ldots, d$. Intuitively speaking, instead of choosing $d$ random points in $\mathbb{R}^{d}$, we first choose a random hyperplane and then, in a second step, choose $d$ random points in this hyperplane. More precisely, the corresponding transformation involves a Jacobian of the form $\left[x_{1}, \ldots, x_{d}\right]$.

In this paper, we need an analogous formula for two sets of points. The points $x_{1}, \ldots, x_{2 d-k}$ determine two hyperplanes, $H_{1}$ and $H_{2}$, which are the affine spans of $x_{1}, \ldots, x_{d}$ and 
$x_{d-k+1}, \ldots, x_{2 d-k}$, respectively. The following formula of Blaschke-Petkantschin type relates the $d$-dimensional volume elements $\mathrm{d} \lambda_{d}\left(x_{j}\right), j=1, \ldots, 2 d-k$, to the differentials $\mathrm{d} \bar{\mu}\left(H_{1}\right)$ and $\mathrm{d} \bar{\mu}\left(H_{2}\right)$ of the hyperplanes $H_{1}$ and $H_{2}$; to the $(d-1)$-dimensional volume elements $\mathrm{d} \lambda_{H_{1}}\left(x_{j}\right)$ and $\mathrm{d} \lambda_{H_{2}}\left(x_{l}\right)$ of points $x_{j}, j=1, \ldots, d-k$, and $x_{l}, l=d+1, \ldots, 2 d-k$ (that are contained in exactly one hyperplane); and to the $(d-2)$-dimensional volume elements $\mathrm{d} \lambda_{H_{1} \cap H_{2}}\left(x_{j}\right)$ of points $x_{j}, j=d-k+1, \ldots, d$ (that are contained in both hyperplanes). Again, such a transformation involves a Jacobian that takes into account the angle between the hyperplanes; this angle is defined as the angle between the normal vectors of the hyperplanes. Since we only consider the sine of this angle, the orientations of the normal vectors need not be specified.

Lemma 3.1. Let $0 \leq k \leq d-1$ and let $g:\left(\mathbb{R}^{d}\right)^{2 d-k} \rightarrow \mathbb{R}$ be a nonnegative measurable function. Then,

$$
\begin{aligned}
\int_{\mathbb{R}^{d}} \cdots \int_{\mathbb{R}^{d}} g\left(x_{1}, \ldots, x_{2 d-k}\right) \prod_{j=1}^{2 d-k} \mathrm{~d} \lambda_{d}\left(x_{j}\right) \\
=\Gamma(d)^{2} \int_{\mathscr{H}_{d-1}^{d}} \int_{\mathscr{H}_{d-1}^{d}} \int_{H_{1}} \cdots \int_{H_{1}} \int_{H_{1} \cap H_{2}} \cdots \int_{H_{1} \cap H_{2}} \int_{H_{2}} \cdots \int_{H_{2}} g\left(x_{1}, \ldots, x_{2 d-k}\right) \\
\times \lambda_{H_{1}}\left(\left[x_{1}, \ldots, x_{d}\right]\right) \lambda_{H_{2}}\left(\left[x_{d-k+1}, \ldots, x_{2 d-k}\right]\right)(\sin \varphi)^{-k} \\
\times \prod_{j=d+1}^{2 d-k} \mathrm{~d} \lambda_{H_{2}}\left(x_{j}\right) \prod_{j=d-k+1}^{d} \mathrm{~d} \lambda_{H_{1} \cap H_{2}}\left(x_{j}\right) \prod_{j=1}^{d-k} \mathrm{~d} \lambda_{H_{1}}\left(x_{j}\right) \mathrm{d} \bar{\mu}\left(H_{1}\right) \mathrm{d} \bar{\mu}\left(H_{2}\right),
\end{aligned}
$$

where $\sin \varphi$ denotes the sine of the angle between $H_{1}$ and $H_{2}$.

Proof. The Blaschke-Petkantschin formula, applied to $x_{1}, \ldots, x_{d}$, and Fubini's theorem show that

$$
\begin{aligned}
\int_{\mathbb{R}^{d}} \ldots \int_{\mathbb{R}^{d}} g\left(x_{1}, \ldots, x_{2 d-k}\right) \prod_{j=1}^{2 d-k} \mathrm{~d} \lambda_{d}\left(x_{j}\right) \\
=\Gamma(d) \int_{\mathscr{H}_{d-1}^{d}} \int_{H_{1}} \cdots \int_{H_{1}} \int_{\mathbb{R}^{d}} \cdots \int_{\mathbb{R}^{d}} g\left(x_{1}, \ldots, x_{2 d-k}\right) \lambda_{H_{1}}\left(\left[x_{1}, \ldots, x_{d}\right]\right) \\
\times \prod_{j=d+1}^{2 d-k} \mathrm{~d} \lambda_{d}\left(x_{j}\right) \prod_{j=1}^{d} \mathrm{~d} \lambda_{H_{1}}\left(x_{j}\right) \mathrm{d} \bar{\mu}\left(H_{1}\right) .
\end{aligned}
$$

We fix $H_{1}$ and let

$$
\tau(f)=\int_{H_{1}} \cdots \int_{H_{1}} \int_{\mathbb{R}^{d}} \cdots \int_{\mathbb{R}^{d}} f\left(x_{d-k+1}, \ldots, x_{2 d-k}\right) \prod_{j=d+1}^{2 d-k} \mathrm{~d} \lambda_{d}\left(x_{j}\right) \prod_{j=d-k+1}^{d} \mathrm{~d} \lambda_{H_{1}}\left(x_{j}\right)
$$

for nonnegative measurable functions $f:\left(\mathbb{R}^{d}\right)^{d} \rightarrow \mathbb{R}$.

An essential ingredient of our proof is a special case of a generalized linear BlaschkePetkantschin formula due to Jensen and Kiêu [12] (see also [11, Theorem 5.6, p. 135]). For all 
nonnegative measurable functions $h$ and for a (fixed) hyperplane $H$, we have

$$
\begin{aligned}
\int_{H} \cdots \int_{H} \int_{\mathbb{R}^{d}} \cdots \int_{\mathbb{R}^{d}} h\left(y_{1}, \ldots, y_{d-1}\right) \prod_{j=k+1}^{d-1} \mathrm{~d} \lambda_{d}\left(y_{j}\right) \prod_{j=1}^{k} \mathrm{~d} \lambda_{H}\left(y_{j}\right) \\
=\Gamma(d) \int_{\mathcal{L}_{d-1}^{d}} \int_{H \cap L} \cdots \int_{H \cap L} \int_{L} \cdots \int_{L} h\left(y_{1}, \ldots, y_{d-1}\right) \\
\quad \times \lambda_{L}\left(\left[0, y_{1}, \ldots, y_{d-1}\right]\right)(\sin \varphi)^{-k} \prod_{j=k+1}^{d-1} \mathrm{~d} \lambda_{L}\left(y_{j}\right) \prod_{j=1}^{k} \mathrm{~d} \lambda_{H \cap L}\left(y_{j}\right) \mathrm{d} \bar{\nu}(L),
\end{aligned}
$$

where $\varphi$ denotes the angle between $H$ and $L$, and $\bar{v}$ is the rotation-invariant Haar measure on the Grassmannian $\mathcal{L}_{d-1}^{d}$ of $(d-1)$-dimensional linear subspaces of $\mathbb{R}^{d}$ with total measure $\frac{1}{2} d \kappa_{d}$.

Using a standard argument (see, e.g. [26, Section 6.1]) this immediately gives a generalized affine Blaschke-Petkantschin formula for $\mathcal{I}(f)$. Let

$$
H=H_{1}-x_{2 d-k} \quad \text { and } \quad x_{d-k+j}-x_{2 d-k}=y_{j}
$$

then

$$
\begin{aligned}
& x(f)= \int_{\mathbb{R}^{d}} \int_{H} \ldots \int_{H} \int_{\mathbb{R}^{d}} \ldots \int_{\mathbb{R}^{d}} f\left(y_{1}+x_{2 d-k}, \ldots, y_{d-1}+x_{2 d-k}, x_{2 d-k}\right) \\
& \times \prod_{j=k+1}^{d-1} \mathrm{~d} \lambda_{d}\left(y_{j}\right) \prod_{j=1}^{k} \mathrm{~d} \lambda_{H}\left(y_{j}\right) \mathrm{d} \lambda_{d}\left(x_{2 d-k}\right) \\
&=\Gamma(d) \int_{\mathbb{R}^{d}} \int_{\mathscr{L}_{d-1}^{d}} \int_{H \cap L} \ldots \int_{H \cap L} \int_{L} \ldots \int_{L} f\left(y_{1}+x_{2 d-k}, \ldots, y_{d-1}+x_{2 d-k}, x_{2 d-k}\right) \\
& \times \lambda_{L}\left(\left[0, y_{1}, \ldots, y_{d-1}\right]\right)(\sin \varphi)^{-k} \\
& \times \prod_{j=k+1}^{d-1} \mathrm{~d} \lambda_{L}\left(y_{j}\right) \prod_{j=1}^{k} \mathrm{~d} \lambda_{H \cap L}\left(y_{j}\right) \mathrm{d} \bar{v}(L) \mathrm{d} \lambda_{d}\left(x_{2 d-k}\right) \\
&=\Gamma(d) \int_{\mathcal{H}_{d-1}^{d}} \int_{H_{1} \cap H_{2}} \ldots \int_{H_{1} \cap H_{2}} \int_{H_{2}} \ldots \int_{H_{2}} f\left(x_{d-k+1}, \ldots, x_{2 d-k}\right) \\
& \times \lambda_{H_{2}}\left(\left[x_{d-k+1}, \ldots, x_{2 d-k}\right]\right)(\sin \varphi)^{-k} \\
& \quad \times \prod_{j=d+1}^{2 d-k} \mathrm{~d} \lambda_{H_{2}}\left(x_{j}\right) \prod_{j=d-k+1}^{d} \mathrm{~d} \lambda_{H_{1} \cap H_{2}}\left(x_{j}\right) \mathrm{d} \bar{\mu}\left(H_{2}\right) .
\end{aligned}
$$

The proof of the lemma follows easily, by setting

$$
f\left(x_{d-k+1}, \ldots, x_{2 d-k}\right)=\Gamma(d) g\left(x_{1}, \ldots, x_{2 d-k}\right) \lambda_{H_{1}}\left(\left[x_{1}, \ldots, x_{d}\right]\right)
$$

for fixed $x_{1}, \ldots, x_{d-k}$. 


\section{Some auxiliary estimates}

Assume that $X$ follows the standard normal distribution $N\left(0, \frac{1}{2} I_{d}\right)$ in $\mathbb{R}^{d}$ with covariance matrix $\frac{1}{2} I_{d}$. Denote by $\phi_{d}(x)$, or simply $\phi(x)$ if $d=1$, the density of the standard normal distribution. The one-dimensional normal distribution $N\left(0, \frac{1}{2}\right)$ is given by

$$
\Phi(z):=\int_{-\infty}^{z} \phi(x) \mathrm{d} x=\frac{1}{\sqrt{\pi}} \int_{-\infty}^{z} \mathrm{e}^{-x^{2}} \mathrm{~d} x, \quad z \in \mathbb{R} .
$$

The corresponding measures, having these functions as densities with respect to the appropriate Lebesgue measure, will be denoted by $\mathrm{d} \phi_{d}(x)$ instead of $\phi_{d}(x) \mathrm{d} x$, etc.

We will repeatedly use the following asymptotic expansions concerning the density of the normal distribution.

Lemma 4.1. Let $j \geq 0$ and $\gamma>0$. Then, as $h_{1} \rightarrow \infty$,

$$
\int_{h_{1}}^{\infty}\left(h_{2}-h_{1}\right)^{j} \phi\left(h_{2}\right)^{\gamma} \mathrm{d} h_{2}=\frac{\Gamma(j+1)}{(2 \gamma)^{j+1}} h_{1}^{-(j+1)} \phi\left(h_{1}\right)^{\gamma}\left(1+O\left(h_{1}^{-2}\right)\right) .
$$

Lemma 4.2. For $n \in \mathbb{N}, \alpha, \beta \in \mathbb{R}$, and $\gamma>0$,

$$
\int_{1}^{\infty} \Phi\left(h_{1}\right)^{n-\alpha} h_{1}^{\beta} \phi\left(h_{1}\right)^{\gamma} \mathrm{d} h_{1}=\Gamma(\gamma) 2^{\gamma-1} n^{-\gamma}(\ln n)^{(\beta+\gamma-1) / 2}(1+o(1))
$$

as $n \rightarrow \infty$.

The proof of Lemma 4.1 is immediate, by the substitution $t=2 \gamma h_{1}\left(h_{2}-h_{1}\right)$. The proof of Lemma 4.2 is a direct generalization of an argument given in [1].

We now provide two useful estimates, which will be needed later.

Lemma 4.3. Let $j, l \geq 0$ and $\gamma>0$. Then there exists a constant $c>0$, depending only on $j, l$, and $\gamma$, such that, for $h_{1} \geq 1$,

$$
\int_{h_{1}}^{\infty} \int_{0}^{\pi}\left(h_{2}-h_{1}\right)^{j} \phi\left(h_{2}\right)^{\gamma} \phi\left(\frac{1}{2} h_{1} \sin \varphi\right)(\sin \varphi)^{l} \mathrm{~d} \varphi \mathrm{d} h_{2} \leq c h_{1}^{-(j+l+2)} \phi\left(h_{1}\right)^{\gamma} .
$$

Proof. Since the sine function is symmetric about $\frac{1}{2} \pi$, by Fubini's theorem and Lemma 4.1 we obtain

$$
\begin{aligned}
& \int_{h_{1}}^{\infty} \int_{0}^{\pi}\left(h_{2}-h_{1}\right)^{j} \phi\left(h_{2}\right)^{\gamma} \phi\left(\frac{1}{2} h_{1} \sin \varphi\right)(\sin \varphi)^{l} \mathrm{~d} \varphi \mathrm{d} h_{2} \\
& \quad=2 \int_{h_{1}}^{\infty}\left(h_{2}-h_{1}\right)^{j} \phi\left(h_{2}\right)^{\gamma} \mathrm{d} h_{2} \int_{0}^{\pi / 2} \phi\left(\frac{1}{2} h_{1} \sin \varphi\right)(\sin \varphi)^{l} \mathrm{~d} \varphi \\
& \quad \leq 2 c_{1} h_{1}^{-(j+1)} \phi\left(h_{1}\right)^{\gamma} \int_{0}^{\pi / 2} \phi\left(\frac{h_{1} \varphi}{\pi}\right) \varphi^{l} \mathrm{~d} \varphi,
\end{aligned}
$$

where the fact that $(2 / \pi) \varphi \leq \sin \varphi \leq \varphi$ for $0 \leq \varphi \leq \frac{1}{2} \pi$ was used in the last step. Here, the constant $c_{1}$ depends only on $j$ and $\gamma$. Making the substitution $h_{1} \varphi=t$, we obtain

$$
\int_{0}^{\pi / 2} \phi\left(\frac{h_{1} \varphi}{\pi}\right) \varphi^{l} \mathrm{~d} \varphi \leq h_{1}^{-(l+1)} \int_{0}^{\infty} \phi\left(\frac{t}{\pi}\right) t^{l} \mathrm{~d} t \leq c_{2} h_{1}^{-(l+1)},
$$

where $c_{2}$ is a constant depending only on $l$. Thus, the assertion follows. 
Lemma 4.4. Let $j, l \geq 0$ and $\gamma>0$. Then there exists a constant $c>0$, depending only on $j, l$, and $\gamma$, such that, for $h_{1} \geq 1$,

$$
\int_{h_{1}}^{\infty} \int_{0}^{\pi}\left(h_{2}-h_{1}\right)^{j} \phi\left(h_{2}\right)^{\gamma} \phi\left(\frac{h_{1}-h_{2} \cos \varphi}{2 \sin \varphi}\right)(\sin \varphi)^{l-1} \mathrm{~d} \varphi \mathrm{d} h_{2} \leq c h_{1}^{-(j+l+1)} \phi\left(h_{1}\right)^{\gamma} .
$$

Proof. We will use Fubini's theorem repeatedly and let $c_{1}, c_{2}, \ldots$ denote constants depending only on $j, l$, and $\gamma$. Then, first making the substitutions (for fixed $h_{2}$ )

$$
u=\frac{h_{1}-h_{2} \cos \varphi}{\sin \varphi}, \quad \mathrm{d} u=\frac{h_{2}-h_{1} \cos \varphi}{\sin ^{2} \varphi} \mathrm{d} \varphi=\sqrt{u^{2}+h_{2}^{2}-h_{1}^{2}} \sin ^{-1} \varphi \mathrm{d} \varphi
$$

with

$$
\sin \varphi=\frac{1}{u^{2}+h_{2}^{2}}\left(h_{1} u+h_{2} \sqrt{u^{2}+h_{2}^{2}-h_{1}^{2}}\right),
$$

and then defining $s$ such that $h_{2}=h_{1}+s^{2} / 2 h_{1}$, we find that

$$
\begin{aligned}
& \int_{h_{1}}^{\infty} \int_{0}^{\pi}\left(h_{2}-h_{1}\right)^{j} \phi\left(h_{2}\right)^{\gamma} \phi\left(\frac{h_{1}-h_{2} \cos \varphi}{2 \sin \varphi}\right)(\sin \varphi)^{l-1} \mathrm{~d} \varphi \mathrm{d} h_{2} \\
& \quad=\int_{h_{1}}^{\infty} \int_{-\infty}^{\infty}\left(h_{2}-h_{1}\right)^{j} \phi\left(h_{2}\right)^{\gamma} \phi\left(\frac{1}{2} u\right) \frac{\left(h_{1} u+h_{2} \sqrt{u^{2}+h_{2}^{2}-h_{1}^{2}}\right)^{l}}{\sqrt{u^{2}+h_{2}^{2}-h_{1}^{2}}\left(u^{2}+h_{2}^{2}\right)^{l}} \mathrm{~d} u \mathrm{~d} h_{2} \\
& \leq c_{1} h_{1}^{-j} \phi\left(h_{1}\right)^{\gamma} \int_{-\infty}^{\infty} \int_{0}^{\infty} \phi(s)^{\gamma} \phi\left(\frac{s^{2}}{2 h_{1}}\right)^{\gamma} \phi\left(\frac{1}{2} u\right) s^{2 j} h_{1}^{-2 l} \\
& \quad \times \frac{\left(h_{1} u+\left(h_{1}+s^{2} / 2 h_{1}\right) \sqrt{u^{2}+s^{2}+s^{4} / 4 h_{1}^{2}}\right)^{l}}{h_{1}} \frac{u^{2}+s^{2}+s^{4} / 4 h_{1}^{2}}{h_{1}} \mathrm{~d} u \\
& \leq c_{2} h_{1}^{-(j+l+1)} \phi\left(h_{1}\right)^{\gamma} \int_{-\infty}^{\infty} \int_{0}^{\infty} \phi(s)^{\gamma} \phi\left(\frac{1}{2} u\right) s^{2 j} \frac{\sqrt{u^{2}+s^{2}+s^{4} / 4 h_{1}^{2}}}{s^{4}} \\
& { }^{l}
\end{aligned}
$$

since the last double integral is finite.

\section{Reduction of Theorem 1.1 to Theorem 1.3}

In this section, we prove that Theorem 1.1 can be deduced from Theorem 1.3. The method of proof is a combination of the Efron-Stein jackknife inequality [4] and the fact that a Gaussian polytope is simple with probability 1 . For random polytopes under a different distribution (for the convex hull of random points chosen in a given smooth convex set) this method was developed in [20]; see also [21]. Although the present approach is similar in the sense that the Efron-Stein jackknife inequality is used, it turns out that the computations needed in the case 
of Gaussian polytopes are much more involved. In particular, we use the facts that the normal distribution is rotationally invariant and the image measure of a Gaussian measure under an orthogonal projection is itself a Gaussian measure.

If $S \equiv S\left(Y_{1}, \ldots, Y_{n}\right)$ is any real symmetric function of the independent, identically distributed random vectors $Y_{j}, 1 \leq j<\infty$, we let

$$
\begin{aligned}
S_{i} & =S\left(Y_{1}, \ldots, Y_{i-1}, Y_{i+1}, \ldots, Y_{n+1}\right), \\
S_{(\cdot)} & =(n+1)^{-1} \sum_{i=1}^{n+1} S_{i} .
\end{aligned}
$$

The Efron-Stein jackknife inequality then states that

$$
\operatorname{var} S \leq \mathrm{E} \sum_{i=1}^{n+1}\left(S_{i}-S_{(\cdot)}\right)^{2}=(n+1) \mathrm{E}\left(S_{n+1}-S_{(\cdot)}\right)^{2} .
$$

Note that the right-hand side does not decrease if $S_{(\cdot)}$ is replaced by any other function of $Y_{1}, \ldots, Y_{n+1}$.

We apply this inequality to the random variable $f\left(P_{n}\right)$, where $f(\cdot)$ is a measurable function of convex polytopes. Then

$$
S=f\left(\left[X_{1}, \ldots, X_{n}\right]\right)=f\left(P_{n}\right),
$$

and we replace $S_{(\cdot)}$ by $f\left(P_{n+1}\right)$, which is a function of the convex hull of $P_{n}$ and a further random point $X_{n+1}$. The Efron-Stein jackknife inequality then yields

$$
\operatorname{var} f\left(P_{n}\right) \leq(n+1) \mathrm{E}\left(f\left(P_{n}\right)-f\left(P_{n+1}\right)\right)^{2} .
$$

In the case that $f(\cdot)$ is the number of $i$-faces of $P_{n}$, we obtain

$$
\operatorname{var} f_{i}\left(P_{n}\right) \leq(n+1) \mathrm{E}\left(f_{i}\left(P_{n+1}\right)-f_{i}\left(P_{n}\right)\right)^{2} .
$$

Let $P_{n}$ be fixed and choose the additional random point $X_{n+1}$. If $X_{n+1}$ is contained in $P_{n}$ then the random variable $f_{i}\left(P_{n+1}\right)-f_{i}\left(P_{n}\right)$ equals 0 . If $X_{n+1} \notin P_{n}$ then the relative interior of some of the $i$-dimensional faces of $P_{n}$ is contained in the interior of $\left[P_{n}, X_{n+1}\right]$ (let $f_{i}^{-}\left(X_{n+1}\right)$ be the number of such faces), and some of the $i$-dimensional faces of $\left[P_{n}, X_{n+1}\right]$ are not contained in $P_{n}$ (let $f_{i}^{+}\left(X_{n+1}\right)$ be the number of such faces). We then have

$$
\left|f_{i}\left(\left[P_{n}, X_{n+1}\right]\right)-f_{i}\left(P_{n}\right)\right|=\left|f_{i}^{+}\left(X_{n+1}\right)-f_{i}^{-}\left(X_{n+1}\right)\right| \leq f_{i}^{+}\left(X_{n+1}\right)+f_{i}^{-}\left(X_{n+1}\right) .
$$

Since $P_{n}$ is simplicial with probability 1 , this number can easily be estimated in terms of $F_{n}\left(X_{n+1}\right)$, the number of facets of $P_{n}$ that can be seen from $X_{n+1}$. Here, $F_{n}\left(X_{n+1}\right)=0$ if $X_{n+1}$ is contained in $P_{n}$, and if $X_{n+1} \notin P_{n}$ then $F_{n}\left(X_{n+1}\right)>0$ is the number of facets of $P_{n}$ that are - up to $(d-2)$-dimensional faces - contained in the interior of the convex hull of $P_{n}$ and $X_{n+1}$. Now, each $i$-dimensional 'new' face of $\left[P_{n}, X_{n+1}\right]$, not contained in $P_{n}$, is the convex hull of $X_{n+1}$ and an $(i-1)$-dimensional face of $P_{n}$. Since this $(i-1)$-dimensional face is also a face of a facet of $P_{n}$ that can be seen from $X_{n+1}$, and each facet is a simplex, we obtain

$$
f_{i}^{+}\left(X_{n+1}\right) \leq\left(\begin{array}{l}
d \\
i
\end{array}\right) F_{n}\left(X_{n+1}\right) .
$$


On the other hand, each $i$-dimensional face of $P_{n}$ that is - up to $(i-1)$-dimensional faces - contained in the interior of $\left[P_{n}, X_{n+1}\right]$ is also a face of a facet contained in the interior of $\left[P_{n}, X_{n+1}\right]$. Hence,

$$
f_{i}^{-}\left(X_{n+1}\right) \leq\left(\begin{array}{c}
d \\
i+1
\end{array}\right) F_{n}\left(X_{n+1}\right),
$$

and combining these estimates proves that

$$
\mathrm{E}\left(f_{i}\left(P_{n+1}\right)-f_{i}\left(P_{n}\right)\right)^{2} \leq\left(\begin{array}{c}
d+1 \\
i+1
\end{array}\right)^{2} \mathrm{E} F_{n}\left(X_{n+1}\right)^{2} .
$$

Thus, each estimate for the second moment of $F_{n}\left(X_{n+1}\right)$ yields an estimate for var $f_{i}\left(P_{n}\right)$ and, hence, Theorem 1.1 follows from Theorem 1.3.

\section{Proof of Theorem 1.3}

\subsection{Asymptotic expansion of the expectation}

We start with the proof of the first part of Theorem 1.3. The case $d=1$ is included by correct interpretation of the subsequent arguments. Choose $n+1$ independent, normally distributed random points $X_{1}, \ldots, X_{n}, X$ in $\mathbb{R}^{d}$. The convex hull of the first $n$ points is a Gaussian polytope $P_{n}$, which is simplicial with probability 1 . For $I \subset\{1, \ldots, n\}$ with $|I|=d$, denote by $F_{I}$ the convex hull of $\left\{X_{i}, i \in I\right\}$, which is a $(d-1)$-dimensional simplex. The affine hull of $F_{I}$ is denoted by $H\left(F_{I}\right)$. With probability 1 , this affine hull is a hyperplane that divides $\mathbb{R}^{d}$ into two (closed) half-spaces. The half-space that contains the origin will be denoted by $H_{0}\left(F_{I}\right)$, and the other by $H_{+}\left(F_{I}\right)$. (The origin is contained in exactly one half-space with probability 1 ). In the following, we want to assume that $P_{n}$ contains the origin. This happens with high probability since, by Wendel's theorem [28],

$$
\mathrm{P}\left(0 \notin P_{n}\right)=O\left(n^{d} 2^{-n}\right)
$$

and, thus, the condition that $P_{n}$ contains the origin can be enforced by adding a suitable error term. Moreover, we can also assume that the points $X_{1}, \ldots, X_{n}, X$ are in general relative position (i.e. any subset of at most $d+1$ of these random points is affinely independent).

We are interested in the number of facets of $P_{n}$ that can be seen from the additional random point $X \notin P_{n}$. Denote the set of these facets by $\mathcal{F}_{n}(X)$, i.e.

$$
\begin{aligned}
\mathcal{F}_{n}(X) & =: \mathcal{F}\left(X_{1}, \ldots, X_{n} ; X\right) \\
& =\left\{F_{I}: P_{n} \subset H_{0}\left(F_{I}\right), X \in H_{+}\left(F_{I}\right), I \subset\{1, \ldots, n\},|I|=d\right\} .
\end{aligned}
$$

Here, we can define $\mathcal{F}_{n}(X)$ as the empty set if the origin is not contained in the interior of $P_{n}$ or if the random points are not in general relative position. Similar definitions can be given for deterministic points $x_{1}, \ldots, x_{n}, x$ in general relative position such that the convex hull of $x_{1}, \ldots, x_{n}$ contains the origin. When applying Wendel's theorem in considering $\mathrm{E} F_{n}(X)$, the error term is of the order $n^{2 d} 2^{-n}=O\left(c^{-n}\right)$, with a suitable constant $c>1$, since $F_{n}(X)$ is bounded by $\left(\begin{array}{l}n \\ d\end{array}\right)$. Using this, we have

$$
\mathrm{E} F_{n}(X)=\int_{\mathbb{R}^{d}} \cdots \int_{\mathbb{R}^{d}} \sum \mathbf{1}_{\left\{F_{I} \in \mathcal{F}_{n}(x)\right\}} \prod_{j=1}^{n} \mathrm{~d} \phi_{d}\left(x_{j}\right) \mathrm{d} \phi_{d}(x)+O\left(c^{-n}\right),
$$


where the summation extends over all subsets $I \subset\{1, \ldots, n\}$ with $|I|=d$. Denote by $F_{1}$ the convex hull of $x_{1}, \ldots, x_{d}$. Then,

$$
\mathrm{E} F_{n}(X)=\left(\begin{array}{l}
n \\
d
\end{array}\right) \int_{\mathbb{R}^{d}} \cdots \int_{\mathbb{R}^{d}} \mathbf{1}_{\left\{F_{1} \in \mathcal{F}_{n}(x)\right\}} \prod_{j=1}^{n} \mathrm{~d} \phi_{d}\left(x_{j}\right) \mathrm{d} \phi_{d}(x)+O\left(c^{-n}\right) .
$$

The probability content of the half-space $H_{+}\left(F_{1}\right)$ is

$$
\int_{H_{+}\left(F_{1}\right)} \mathrm{d} \phi_{d}(x)=1-\Phi\left(h_{1}\right),
$$

where $h_{1}$ is the distance of $H\left(F_{1}\right)$ to the origin. If $F_{1} \in \mathcal{F}_{n}(X)$ then $X$ is contained in the halfspace $H_{+}\left(F_{1}\right)$ with probability content $1-\Phi\left(h_{1}\right)$, and the random points $X_{j}, j \in\{d+1, \ldots, n\}$, are contained in the half-space $H_{0}\left(F_{1}\right)$ with probability content $\Phi\left(h_{1}\right)$. Hence, we obtain

$$
\mathrm{E} F_{n}(X)=\left(\begin{array}{l}
n \\
d
\end{array}\right) \int_{\mathbb{R}^{d}} \cdots \int_{\mathbb{R}^{d}} \Phi\left(h_{1}\right)^{n-d}\left(1-\Phi\left(h_{1}\right)\right) \prod_{j=1}^{d} \mathrm{~d} \phi_{d}\left(x_{j}\right)+O\left(c^{-n}\right) .
$$

Parametrizing the hyperplane $H\left(F_{1}\right)=: H_{1}$ in terms of the distance $h_{1} \geq 0$ from the origin and its unit normal vector $u_{1}$ in the form $H_{1} \equiv H\left(u_{1}, h_{1}\right)$, and using the affine BlaschkePetkantschin formula, we find that

$$
\begin{aligned}
& \text { E } F_{n}(X) \\
& =\Gamma(d)\left(\begin{array}{l}
n \\
d
\end{array}\right) \int_{\mathbb{S}^{d-1}} \int_{0}^{\infty} \Phi\left(h_{1}\right)^{n-d}\left(1-\Phi\left(h_{1}\right)\right) \\
& \times\left\{\int_{H_{1}} \cdots \int_{H_{1}} \lambda_{H_{1}}\left(\left[x_{1}, \ldots, x_{d}\right]\right) \prod_{j=1}^{d}\left(\phi_{d}\left(x_{j}\right) \mathrm{d} \lambda_{H_{1}}\left(x_{j}\right)\right)\right\} \mathrm{d} h_{1} \mathrm{~d} \sigma\left(u_{1}\right) \\
& +O\left(c^{-n}\right) \text {. }
\end{aligned}
$$

The inner integral (in brackets) is the product of $\phi\left(h_{1}\right)^{d}$ and the expected volume

$$
\mathrm{E} V_{d-1}\left(P_{d}^{(d-1)}\right)
$$

of a random $(d-1)$-dimensional Gaussian simplex in $\mathbb{R}^{d-1}$. Therefore,

$$
\begin{aligned}
\mathrm{E} F_{n}(X)= & \Gamma(d)\left(\begin{array}{l}
n \\
d
\end{array}\right) \mathrm{E} V_{d-1}\left(P_{d}^{(d-1)}\right) \int_{\mathbb{S}^{d-1}} \int_{0}^{\infty} \Phi\left(h_{1}\right)^{n-d}\left(1-\Phi\left(h_{1}\right)\right) \phi\left(h_{1}\right)^{d} \mathrm{~d} h_{1} \mathrm{~d} \sigma\left(u_{1}\right) \\
& +O\left(c^{-n}\right)
\end{aligned}
$$

The expected volume of a random Gaussian simplex was computed explicitly by Miles [17]. It now follows from Lemma 4.1 and Lemma 4.2 that

$$
\mathrm{E} F_{n}(X)=2^{d-1} \kappa_{d} \Gamma(d+1) \mathrm{E} V_{d-1}\left(P_{d}^{(d-1)}\right) n^{-1}(\ln n)^{(d-1) / 2}(1+o(1)) .
$$

This proves the first part of Theorem 1.3. 


\subsection{Estimate of the variance}

The main part of the proof is devoted to estimating the second moment of $F_{n}(X)$. In the case $d=1$, we have $F_{n}(X)=F_{n}(X)^{2}$, whence the assertion follows. Now let $d \geq 2$.

As in (6.1), we have

$$
\mathrm{E} F_{n}(X)^{2}=\int_{\mathbb{R}^{d}} \cdots \int_{\mathbb{R}^{d}}\left(\sum_{I} \mathbf{1}_{\left\{F_{I} \in \mathcal{F}_{n}(x)\right\}}\right)^{2} \prod_{j=1}^{n} \mathrm{~d} \phi_{d}\left(x_{j}\right) \mathrm{d} \phi_{d}(x)+O\left(c^{-n}\right),
$$

with some $c>1$. The summation extends over all subsets $I \subset\{1, \ldots, n\}$ with $|I|=d$. We expand the integrand to obtain

$$
\mathrm{E} F_{n}(X)^{2}=\sum_{I} \sum_{J} \int_{\mathbb{R}^{d}} \cdots \int_{\mathbb{R}^{d}} \mathbf{1}_{\left\{F_{I}, F_{J} \in \mathcal{F}_{n}(x)\right\}} \prod_{j=1}^{n} \mathrm{~d} \phi_{d}\left(x_{j}\right) \mathrm{d} \phi_{d}(x)+O\left(c^{-n}\right),
$$

where the summation extends over all subsets $I, J \subset\{1, \ldots, n\}$ with $|I|=|J|=d$. If we fix the number $k=|I \cap J| \in\{0, \ldots, d\}$, then the corresponding term in (6.3) depends only on $k$ and not on the particular choice of $I$ and $J$. For a given $k \in\{0, \ldots, d\}$, we let

$$
\begin{aligned}
F_{1} & =\left[X_{1}, \ldots, X_{d}\right], \\
F_{2}^{(k)} & =\left[X_{d-k+1}, \ldots, X_{2 d-k}\right] .
\end{aligned}
$$

Note that for $k=d$ we have $F_{1}=F_{2}^{(d)}$. Hence, E $F_{n}(X)^{2}$ can be rewritten as $\mathrm{E} F_{n}(X)^{2}=\sum_{k=0}^{d}\left(\begin{array}{l}n \\ d\end{array}\right)\left(\begin{array}{l}d \\ k\end{array}\right)\left(\begin{array}{l}n-d \\ d-k\end{array}\right) \int_{\mathbb{R}^{d}} \cdots \int_{\mathbb{R}^{d}} \mathbf{1}_{\left\{F_{1}, F_{2}^{(k)} \in \mathcal{F}_{n}(x)\right\}} \prod_{j=1}^{n} \mathrm{~d} \phi_{d}\left(x_{j}\right) \mathrm{d} \phi_{d}(x)+O\left(c^{-n}\right)$.

The summand corresponding to $k=d$ is just $\mathrm{E} F_{n}(X)$ and, thus, (6.2) yields

$$
\begin{aligned}
\mathrm{E} F_{n}(X)^{2} \leq & c_{1} \sum_{k=0}^{d-1} n^{2 d-k} \int_{\mathbb{R}^{d}} \cdots \int_{\mathbb{R}^{d}} \mathbf{1}_{\left\{F_{1}, F_{2}^{(k)} \in \mathcal{F}_{n}(x)\right\}} \prod_{j=1}^{n} \mathrm{~d} \phi_{d}\left(x_{j}\right) \mathrm{d} \phi_{d}(x) \\
& +O\left(n^{-1}(\ln n)^{(d-1) / 2}\right) .
\end{aligned}
$$

Here, and in the following, $c_{1}, c_{2}, \ldots$ denote constants that are independent of $n$. The summand corresponding to $k=d$ also yields the asserted lower bound for $\mathrm{E} F_{n}(X)^{2}$.

Let $h_{1}$ and $h_{2}$ be the distances to the origin and $u_{1}$ and $u_{2}$ the unit normal vectors of $H\left(F_{1}\right)$ and $H\left(F_{2}^{(k)}\right)$, respectively, such that

$$
H\left(F_{1}\right)=H\left(u_{1}, h_{1}\right) \text { and } H\left(F_{2}^{(k)}\right)=H\left(u_{2}, h_{2}\right) .
$$

Since the integrand is symmetric in $F_{1}$ and $F_{2}^{(k)}$, we restrict our integration to the range $h_{1} \leq h_{2}$. Thus, we obtain

$$
\begin{aligned}
\mathrm{E} F_{n}(X)^{2} \leq & c_{2} \sum_{k=0}^{d-1} n^{2 d-k} \int_{\mathbb{R}^{d}} \cdots \int_{\mathbb{R}^{d}} \mathbf{1}_{\left\{h_{1} \leq h_{2}\right\}} \mathbf{1}_{\left\{F_{1}, F_{2}^{(k)} \in \mathcal{F}_{n}(x)\right\}} \prod_{j=1}^{n} \mathrm{~d} \phi_{d}\left(x_{j}\right) \mathrm{d} \phi_{d}(x) \\
& +O\left(n^{-1}(\ln n)^{(d-1) / 2}\right) .
\end{aligned}
$$


If $F_{1}, F_{2}^{(k)} \in \mathcal{F}_{n}(x)$ then the points $x_{2 d-k+1}, \ldots, x_{n}$ are contained in $H_{0}\left(F_{1}\right) \cap H_{0}\left(F_{2}^{(k)}\right)$ and the corresponding measure of the set of these points is at most $\Phi\left(h_{1}\right)^{n-2 d+k}$. Moreover, having $F_{1}, F_{2}^{(k)} \in \mathcal{F}_{n}(x)$ implies that $x$ is contained in $H_{+}\left(F_{1}\right) \cap H_{+}\left(F_{2}^{(k)}\right)$. Denote the distance of $H_{+}\left(F_{1}\right) \cap H_{+}\left(F_{2}^{(k)}\right)$ to the origin by $h_{12}$. Then the corresponding measure is at most $1-\Phi\left(h_{12}\right)$. This yields

$$
\begin{aligned}
\mathrm{E} F_{n}(X)^{2} \leq & c_{2} \sum_{k=0}^{d-1} n^{2 d-k} \int_{\mathbb{R}^{d}} \cdots \int_{\mathbb{R}^{d}} \mathbf{1}_{\left\{h_{1} \leq h_{2}\right\}} \Phi\left(h_{1}\right)^{n-2 d+k}\left(1-\Phi\left(h_{12}\right)\right) \prod_{j=1}^{2 d-k} \mathrm{~d} \phi_{d}\left(x_{j}\right) \\
& +O\left(n^{-1}(\ln n)^{(d-1) / 2}\right) .
\end{aligned}
$$

The range of integration can be further reduced to that imposed by $\mathbf{1}_{\left\{1 \leq h_{1} \leq h_{2}\right\}}$, since the additional error term is of order $n^{2 d} \Phi(1)^{n-2 d+k}=O\left(c^{-n}\right)$, with a suitable $c>1$. For $h_{1} \geq 1$, and since $h_{12} \geq h_{1}$, Lemma 4.1 implies that

$$
1-\Phi\left(h_{12}\right) \leq c_{3} h_{1}^{-1} \phi\left(h_{12}\right) .
$$

Therefore, we conclude that

$$
\begin{aligned}
\mathrm{E} F_{n}(X)^{2} \leq & c_{4} \sum_{k=0}^{d-1} n^{2 d-k} \int_{\mathbb{R}^{d}} \cdots \int_{\mathbb{R}^{d}} \mathbf{1}_{\left\{1 \leq h_{1} \leq h_{2}\right\}} \Phi\left(h_{1}\right)^{n-2 d+k} h_{1}^{-1} \phi\left(h_{12}\right) \prod_{j=1}^{2 d-k} \mathrm{~d} \phi_{d}\left(x_{j}\right) \\
& +O\left(n^{-1}(\ln n)^{(d-1) / 2}\right) .
\end{aligned}
$$

To develop an estimate for the integral

$$
\tau_{k}=\int_{\mathbb{R}^{d}} \cdots \int_{\mathbb{R}^{d}} \mathbf{1}_{\left\{1 \leq h_{1} \leq h_{2}\right\}} \Phi\left(h_{1}\right)^{n-2 d+k} h_{1}^{-1} \phi\left(h_{12}\right) \prod_{j=1}^{2 d-k} \mathrm{~d} \phi_{d}\left(x_{j}\right)
$$

in the cases in which $0 \leq k \leq d-1$, we apply the Blaschke-Petkantschin formula (3.1) to obtain

$$
\begin{aligned}
I_{k}=\Gamma(d)^{2} \int_{\mathbb{S}^{d-1}} & \int_{1}^{\infty} \int_{\mathbb{S}^{d}-1} \int_{h_{1}}^{\infty} \Phi\left(h_{1}\right)^{n-2 d+k} h_{1}^{-1} \phi\left(h_{12}\right)(\sin \varphi)^{-k} \\
& \times \mathscr{g}_{k}\left(u_{1}, h_{1}, u_{2}, h_{2}\right) \mathrm{d} h_{2} \mathrm{~d} \sigma\left(u_{2}\right) \mathrm{d} h_{1} \mathrm{~d} \sigma\left(u_{1}\right),
\end{aligned}
$$

with

$$
\begin{aligned}
\mathscr{g}_{k}\left(u_{1}, h_{1}, u_{2}, h_{2}\right)=\int_{H_{1}} & \cdots \int_{H_{1}} \int_{H_{1} \cap H_{2}} \cdots \int_{H_{1} \cap H_{2}} \int_{H_{2}} \cdots \int_{H_{2}} \lambda_{H_{1}}\left(F_{1}\right) \lambda_{H_{2}}\left(F_{2}^{(k)}\right) \\
& \times \prod_{j=1}^{2 d-k} \phi_{d}\left(x_{j}\right) \prod_{j=d+1}^{2 d-k} \mathrm{~d} \lambda_{H_{2}}\left(x_{j}\right) \prod_{j=d-k+1}^{d} \mathrm{~d} \lambda_{H_{1} \cap H_{2}}\left(x_{j}\right) \prod_{j=1}^{d-k} \mathrm{~d} \lambda_{H_{1}}\left(x_{j}\right) .
\end{aligned}
$$

In the following, we distinguish between the cases $k=0$ and $k \in\{1, \ldots, d-1\}$.

First, let $k \in\{1, \ldots, d-1\}$. We denote by $h_{12}^{(d-2)}$ the distance from the origin to the $(d-2)$-dimensional intersection $H_{1} \cap H_{2}$ and define an angle $\varphi^{*} \in\left(0, \frac{1}{2} \pi\right)$ by $h_{1}=h_{2} \cos \varphi^{*}$. 
Then

$$
\begin{array}{ll}
h_{12}=h_{2} \leq h_{12}^{(d-2)} & \text { if } 0 \leq \varphi \leq \varphi^{*}, \\
h_{12}=h_{12}^{(d-2)} & \text { if } \varphi \geq \varphi^{*} .
\end{array}
$$

By $v_{1}$ and $v_{2}$, we denote unit vectors parallel to $H_{1}$ and $H_{2}$, respectively, and orthogonal to $H_{1} \cap H_{2}$. The $(d-1)$-volumes of the simplices $F_{1}$ and $F_{2}^{(k)}$ can be bounded from above by the $(d-2)$-volumes of their projections onto $H_{1} \cap H_{2}$, namely

$$
\operatorname{proj}_{H_{1} \cap H_{2}} F_{1} \text { and } \operatorname{proj}_{H_{1} \cap H_{2}} F_{2}^{(k)} \text {, }
$$

and their heights in the direction orthogonal to $H_{1} \cap H_{2}$. Hence, we obtain

$$
\lambda_{H_{1}}\left(F_{1}\right) \leq \lambda_{H_{1} \cap H_{2}}\left(\operatorname{proj}_{H_{1} \cap H_{2}} F_{1}\right)\left(\max _{j=1, \ldots, d-k}\left|\left\langle x_{j}, v_{1}\right\rangle\right|+\sqrt{\left(h_{12}^{(d-2)}\right)^{2}-h_{1}^{2}}\right),
$$

where we have bounded the height of $F_{1}$ by the sum of the distance between $H_{1} \cap H_{2}$ and $h_{1} u_{1}$, which is $\left(\left(h_{12}^{(d-2)}\right)^{2}-h_{1}^{2}\right)^{1 / 2}$, and the maximal distance from the points $x_{j}$ to the point $h_{1} u_{1}$ in the direction $v_{1}$. Analogously,

$$
\lambda_{H_{2}}\left(F_{2}^{(k)}\right) \leq \lambda_{H_{1} \cap H_{2}}\left(\operatorname{proj}_{H_{1} \cap H_{2}} F_{2}^{(k)}\right)\left(\max _{j=d+1, \ldots, 2 d-k}\left|\left\langle x_{j}, v_{2}\right\rangle\right|+\sqrt{\left(h_{12}^{(d-2)}\right)^{2}-h_{2}^{2}}\right) .
$$

Writing $x_{j} \in H_{1}, j=1, \ldots, d-k$, as the orthogonal sum

$$
x_{j}=h_{1} u_{1}+x_{j}^{1} v_{1}+y_{j},
$$

where $y_{j}$ is contained in the $(d-2)$-dimensional linear subspace parallel to $H_{1} \cap H_{2}$, which we identify with $\mathbb{R}^{d-2}$, we have

$$
\left\langle x_{j}, v_{1}\right\rangle=x_{j}^{1} \quad \text { and } \quad \phi_{d}\left(x_{j}\right)=\phi\left(h_{1}\right) \phi\left(x_{j}^{1}\right) \phi_{d-2}\left(y_{j}\right) .
$$

For the integration with respect $x_{j}^{1}$, we obtain

$$
\int_{\mathbb{R}} \cdots \int_{\mathbb{R}} \max _{j=1, \ldots, d-k}\left|x_{j}^{1}\right| \prod_{j=1}^{d-k}\left(\phi_{d}\left(x_{j}\right) \mathrm{d} x_{j}^{1}\right) \leq c_{5} \prod_{j=1}^{d-k}\left(\phi\left(h_{1}\right) \phi_{d-2}\left(y_{j}\right)\right),
$$

and an analogous result holds for $x_{j} \in H_{2}, j=d+1, \ldots, 2 d-k$. Recall that $h_{1} \leq h_{2}$. This shows that

$$
\begin{aligned}
\mathscr{g}_{k}\left(u_{1}, h_{1}, u_{2}, h_{2}\right) \leq & \left(c_{5}+\sqrt{\left(h_{12}^{(d-2)}\right)^{2}-h_{1}^{2}}\right)^{2} \phi\left(h_{1}\right)^{d-k} \phi\left(h_{2}\right)^{d-k} \phi\left(h_{12}^{(d-2)}\right)^{k} \\
& \times \int_{\mathbb{R}^{d-2}} \cdots \int_{\mathbb{R}^{d-2}} \lambda_{d-2}\left(\left[y_{1}, \ldots, y_{d}\right]\right) \lambda_{d-2}\left(\left[y_{d-k+1}, \ldots, y_{2 d-k}\right]\right) \\
& \times \prod_{j=1}^{2 d-k}\left(\phi_{d-2}\left(y_{j}\right) \mathrm{d} \lambda_{d-2}\left(y_{j}\right)\right) .
\end{aligned}
$$

Using the facts that $(a+b)^{2} \leq 2\left(a^{2}+b^{2}\right)$ and $\left(1+\frac{1}{2} x\right) \mathrm{e}^{-x / 2} \leq 1$ for $a, b \in \mathbb{R}$ and $x \geq 0$, we deduce that

$$
\mathscr{g}_{k}\left(u_{1}, h_{1}, u_{2}, h_{2}\right) \leq c_{6} \phi\left(h_{1}\right)^{d-k+1 / 2} \phi\left(h_{2}\right)^{d-k} \phi\left(h_{12}^{(d-2)}\right)^{k-1 / 2}
$$


and, thus,

$$
\begin{aligned}
I_{k} \leq c_{7} \int_{\mathbb{S} d-1} & \int_{1}^{\infty} \int_{\mathbb{S}^{d-1}} \int_{h_{1}}^{\infty} \Phi\left(h_{1}\right)^{n-2 d+k} h_{1}^{-1} \phi\left(h_{1}\right)^{d-k+1 / 2} \phi\left(h_{2}\right)^{d-k} \\
& \times \phi\left(h_{12}\right) \phi\left(h_{12}^{(d-2)}\right)^{k-1 / 2}(\sin \varphi)^{-k} \mathrm{~d} h_{2} \mathrm{~d} \sigma\left(u_{2}\right) \mathrm{d} h_{1} \mathrm{~d} \sigma\left(u_{1}\right) .
\end{aligned}
$$

Since the integrand is rotation invariant, we may assume that $u_{1}=e_{d}$ (i.e. $u_{1}$ coincides with a basis vector) and, hence, arrive at

$$
\begin{aligned}
\mathfrak{I}_{k} \leq c_{8} \int_{1}^{\infty} & \int_{\mathbb{S}^{d-1}} \int_{h_{1}}^{\infty} \Phi\left(h_{1}\right)^{n-2 d+k} h_{1}^{-1} \phi\left(h_{1}\right)^{d-k+1 / 2} \phi\left(h_{2}\right)^{d-k} \\
& \times \phi\left(h_{12}\right) \phi\left(h_{12}^{(d-2)}\right)^{k-1 / 2}(\sin \varphi)^{-k} \mathrm{~d} h_{2} \mathrm{~d} \sigma\left(u_{2}\right) \mathrm{d} h_{1} .
\end{aligned}
$$

Elementary calculations show that

$$
\left(h_{12}^{(d-2)}\right)^{2}=h_{1}^{2}+\frac{\left(h_{2}-h_{1} \cos \varphi\right)^{2}}{(\sin \varphi)^{2}}=h_{2}^{2}+\frac{\left(h_{1}-h_{2} \cos \varphi\right)^{2}}{(\sin \varphi)^{2}}, \quad \varphi \in(0, \pi) ;
$$

moreover,

$$
\left(h_{12}^{(d-2)}\right)^{2} \geq h_{12}^{2}=h_{2}^{2} \geq h_{1}^{2}\left(1+\frac{1}{4}(\sin \varphi)^{2}\right), \quad \varphi \in\left(0, \varphi^{*}\right],
$$

and

$$
h_{12}^{2}=\left(h_{12}^{(d-2)}\right)^{2} \geq h_{1}^{2}\left(1+\frac{1}{4}(\sin \varphi)^{2}\right), \quad \varphi \in\left[\varphi^{*}, \pi\right) .
$$

We parametrize $u_{2} \in \mathbb{S}^{d-1} \backslash\left\{e_{d},-e_{d}\right\}$ in terms of the angle $\varphi$, between $u_{2}$ and $e_{d}$, and its normalized projection $v$ onto $\mathbb{R}^{d-1}$ (with $v \in \mathbb{S}^{d-1} \cap e_{d}^{\perp}$ and $\varphi \in(0, \pi)$ ). Let $\sigma_{d-2}$ denote the spherical Lebesgue measure on $\mathbb{S}^{d-2}$. We can then estimate the inner double integral in (6.8) by using the fact that $k-\frac{1}{2} \geq \frac{1}{4}$ and Lemma 4.4 :

$$
\begin{aligned}
& \int_{h_{1}}^{\infty} \int_{\mathbb{S}^{d-1}} \phi\left(h_{2}\right)^{d-k} \phi\left(h_{12}\right) \phi\left(h_{12}^{(d-2)}\right)^{k-1 / 2}(\sin \varphi)^{-k} \mathrm{~d} \sigma\left(u_{2}\right) \mathrm{d} h_{2} \\
& \quad \leq c_{9} \phi\left(h_{1}\right)^{k+1 / 2} \int_{h_{1}}^{\infty} \int_{S^{d-2}} \int_{0}^{\pi} \phi\left(h_{2}\right)^{d-k} \phi\left(\frac{h_{1}-h_{2} \cos \varphi}{2 \sin \varphi}\right)(\sin \varphi)^{d-k-2} \mathrm{~d} \varphi \mathrm{d} \sigma_{d-2}(v) \mathrm{d} h_{2} \\
& \leq c_{10} \phi\left(h_{1}\right)^{k+1 / 2} \int_{h_{1}}^{\infty} \int_{0}^{\pi} \phi\left(h_{2}\right)^{d-k} \phi\left(\frac{h_{1}-h_{2} \cos \varphi}{2 \sin \varphi}\right)(\sin \varphi)^{d-k-2} \mathrm{~d} \varphi \mathrm{d} h_{2} \\
& \quad \leq c_{11} h_{1}^{-(d-k)} \phi\left(h_{1}\right)^{d+1 / 2} .
\end{aligned}
$$

Then, for $k \in\{1, \ldots, d-1\}$, we deduce from (6.8) and (6.12) that

$$
\widetilde{I}_{k} \leq c_{12} \int_{1}^{\infty} \Phi\left(h_{1}\right)^{n-2 d+k} h_{1}^{-d+k-1} \phi\left(h_{1}\right)^{2 d+1-k} \mathrm{~d} h_{1} \leq c_{13} n^{-(2 d-k+1)}(\ln n)^{(d-1) / 2}
$$


Finally, we consider the case $k=0$. Using the previous notation and (6.10) and (6.11), from (6.5) and (6.6) we directly obtain

$$
\begin{aligned}
\tau_{0} & \leq c_{14} \int_{\mathbb{S}^{d-1}} \int_{1}^{\infty} \int_{\mathbb{S}^{d-1}} \int_{h_{1}}^{\infty} \Phi\left(h_{1}\right)^{n-2 d} h_{1}{ }^{-1} \phi\left(h_{12}\right) \phi\left(h_{1}\right)^{d} \phi\left(h_{2}\right)^{d} \mathrm{~d} h_{2} \mathrm{~d} \sigma\left(u_{2}\right) \mathrm{d} h_{1} \mathrm{~d} \sigma\left(u_{1}\right) \\
& \leq c_{15} \int_{1}^{\infty} \Phi\left(h_{1}\right)^{n-2 d} h_{1}{ }^{-1} \phi\left(h_{1}\right)^{d+1} \int_{h_{1}}^{\infty} \int_{0}^{\pi} \phi\left(h_{2}\right)^{d} \phi\left(\frac{h_{1} \sin \varphi}{2}\right)(\sin \varphi)^{d-2} \mathrm{~d} \varphi \mathrm{d} h_{2} \mathrm{~d} h_{1} .
\end{aligned}
$$

From Lemma 4.3, we then obtain

$$
\tau_{0} \leq c_{16} \int_{1}^{\infty} \Phi\left(h_{1}\right)^{n-2 d} h_{1}^{-(d+1)} \phi\left(h_{1}\right)^{2 d+1} \mathrm{~d} h_{1} \leq c_{17} n^{-(2 d+1)}(\ln n)^{(d-1) / 2} .
$$

Combining the estimates (6.4), (6.13), and (6.14), we arrive at

$$
\mathrm{E} F_{n}(X)^{2} \leq c_{18} \sum_{k=0}^{d} n^{2 d-k} n^{-2 d+k-1}(\ln n)^{(d-1) / 2} \leq c_{19} n^{-1}(\ln n)^{(d-1) / 2} .
$$

This completes the proof of Theorem 1.3.

\section{Proof of Theorem $\mathbf{1 . 2}$}

The proof is based on arguments similar to those involved in the proofs of Theorems 1.1 and 1.3. Therefore, we use the same notation as before. In particular, $c_{1}, c_{2}, \ldots$ denote constants that depend only on the dimension.

Denote by $v_{i}$ the Haar probability measure on the set $\mathcal{L}_{i}^{d}$ of $i$-dimensional linear subspaces in $\mathbb{R}^{d}$. Kubota's theorem and the rotation invariance of the normal distribution immediately give

$$
\mathrm{E} V_{i}\left(P_{n}\right)=\mathrm{E}\left(c_{d, i} \int_{\mathscr{L}_{i}^{d}} V_{i}\left(\operatorname{proj}_{L} P_{n}\right) \mathrm{d} v_{i}(L)\right)=c_{d, i} \mathrm{E} V_{i}\left(\operatorname{proj}_{L_{0}} P_{n}\right)
$$

(where, recall, $c_{d, i}=\left(\begin{array}{c}d \\ i\end{array}\right) \kappa_{d} / \kappa_{i} \kappa_{d-i}$ ), for an arbitrary $i$-dimensional linear subspace $L_{0}$ that we identify with $\mathbb{R}^{i}$. The projection onto $\mathbb{R}^{i}$ of a Gaussian sample in $\mathbb{R}^{d}$ is itself a Gaussian sample. Hence,

$$
\mathrm{E} V_{i}\left(P_{n}\right)=c_{d, i} \mathrm{E} V_{i}\left(P_{n}^{(i)}\right),
$$

where $P_{n}^{(i)}$ is the convex hull of a Gaussian sample in $\mathbb{R}^{i}$. For the variance, we obtain

$$
\begin{aligned}
\operatorname{var} V_{i}\left(P_{n}\right) & =\mathrm{E}\left(V_{i}\left(P_{n}\right)-\mathrm{E} V_{i}\left(P_{n}\right)\right)^{2} \\
& =\mathrm{E}\left(c_{d, i} \int_{\mathcal{L}_{i}^{d}}\left(V_{i}\left(\operatorname{proj}_{L} P_{n}\right)-\mathrm{E} V_{i}\left(P_{n}^{(i)}\right)\right) \mathrm{d} v_{i}(L)\right)^{2} \\
& \leq c_{d, i}^{2} \mathrm{E}\left(\int_{\mathscr{L}_{i}^{d}}\left(V_{i}\left(\operatorname{proj}_{L} P_{n}\right)-\mathrm{E} V_{i}\left(P_{n}^{(i)}\right)\right)^{2} \mathrm{~d} v_{i}(L)\right)
\end{aligned}
$$

by Hölder's inequality. Thus, Fubini's theorem and rotation invariance imply that

$$
\operatorname{var} V_{i}\left(P_{n}\right) \leq c_{d, i}^{2} \mathrm{E}\left(V_{i}\left(P_{n}^{(i)}\right)-\mathrm{E} V_{i}\left(P_{n}^{(i)}\right)\right)^{2}=c_{d, i}^{2} \operatorname{var} V_{i}\left(P_{n}^{(i)}\right)
$$


Therefore, by the Efron-Stein jackknife inequality (5.1), it suffices to prove that

$$
\operatorname{var} V_{d}\left(P_{n}\right) \leq(n+1) \mathrm{E}\left(V_{d}\left(P_{n+1}\right)-V_{d}\left(P_{n}\right)\right)^{2} \leq c_{1}(\ln n)^{(d-3) / 2}
$$

(i.e. the case $i=d$ ), which then yields the general result.

We will assume that $P_{n}$ contains the origin, which leads to an error term of the order

$$
\left(n^{5 d} 2^{-n}\right)^{1 / 2}=O\left(c^{-n}\right),
$$

with a suitable constant $c>1$. To see this, we can use Cauchy's inequality

$$
\mathrm{E}\left(\left(V_{d}\left(P_{n+1}\right)-V_{d}\left(P_{n}\right)\right)^{2} \mathbf{1}_{\left\{0 \notin P_{n}\right\}}\right) \leq \sqrt{\mathrm{E}\left(V_{d}\left(P_{n+1}\right)-V_{d}\left(P_{n}\right)\right)^{4} \mathrm{P}\left(0 \notin P_{n}\right)}
$$

and Hölder's inequality

$$
\begin{aligned}
\mathrm{E}\left(V_{d}\left(P_{n+1}\right)-V_{d}\left(P_{n}\right)\right)^{4} & \leq \mathrm{E} V_{d}\left(P_{n+1}\right)^{4} \\
& \leq \mathrm{E}\left(\sum_{I} V_{d}\left(\left[0, F_{I}\right]\right)\right)^{4} \\
& \leq\left(\begin{array}{c}
n+1 \\
d
\end{array}\right)^{4} \mathrm{E} V_{d}\left(\left[0, F_{1}\right]\right)^{4} ;
\end{aligned}
$$

here the summation extends over all sets $I \subset\{1, \ldots, n+1\}$ with $|I|=d$. Since Wendel's theorem [28] shows that $\mathrm{P}\left(0 \notin P_{n}\right)=O\left(n^{d} 2^{-n}\right)$ and since $\mathrm{E} V_{d}\left(\left[0, F_{1}\right]\right)^{4}$ is a (finite) constant, the assertion follows. Thus, we obtain

$$
\begin{aligned}
& \mathrm{E}\left(V_{d}\left(P_{n+1}\right)-V_{d}\left(P_{n}\right)\right)^{2}= \mathrm{E}\left(\sum_{I} \mathbf{1}_{\left\{F_{I} \in \mathcal{F}_{n}(X)\right\}} V_{d}\left(\left[F_{I}, X\right]\right)\right)^{2}+O\left(c^{-n}\right) \\
&=2 \sum_{k=0}^{d}\left(\begin{array}{l}
n \\
d
\end{array}\right)\left(\begin{array}{l}
d \\
k
\end{array}\right)\left(\begin{array}{l}
n-d \\
d-k
\end{array}\right) \int_{\mathbb{R}^{d}} \cdots \int_{\mathbb{R}^{d}} \mathbf{1}_{\left\{1 \leq h_{1} \leq h_{2}\right\}} \\
& \times \mathbf{1}_{\left\{F_{1}, F_{2}^{(k)} \in \mathcal{F}_{n}(x)\right\}} V_{d}\left(\left[F_{1}, x\right]\right) V_{d}\left(\left[F_{2}^{(k)}, x\right]\right) \\
& \times \prod_{j=1}^{n} \mathrm{~d} \phi_{d}\left(x_{j}\right) \mathrm{d} \phi_{d}(x)+O\left(c^{-n}\right) \\
& \leq c_{2} \sum_{k=0}^{d} n^{2 d-k} \int_{\mathbb{R}^{d}} \ldots \int_{\mathbb{R}^{d}} \mathbf{1}_{\left\{1 \leq h_{1} \leq h_{2}\right\}} \mathbf{1}_{\left\{F_{1}, F_{2}^{(k)} \in \mathcal{F}_{n}(x)\right\}} \\
& \times V_{d}\left(\left[F_{1}, x\right]\right) V_{d}\left(\left[F_{2}^{(k)}, x\right]\right) \prod_{j=1}^{n} \mathrm{~d} \phi_{d}\left(x_{j}\right) \mathrm{d} \phi_{d}(x)+O\left(c^{-n}\right) .
\end{aligned}
$$

For the integration with respect to $x$, observe that $V_{d}\left(\left[F_{1}, x\right]\right)$ equals $(1 / d) \lambda_{H_{1}}\left(F_{1}\right)$ (which is independent of $x$ ) times the distance $\left\langle x, u_{1}\right\rangle-h_{1}$ between $x$ and $H_{1}=H\left(F_{1}\right)$; a similar assertion holds for $F_{2}^{(k)}$. 
Let us first consider the summand corresponding to $k=d$. In this case, we estimate that

$$
\begin{aligned}
& n^{d} \int_{\mathbb{R}^{d}} \cdots \int_{\mathbb{R}^{d}} \mathbf{1}_{\left\{1 \leq h_{1}\right\}} \mathbf{1}_{\left\{F_{1} \in \mathcal{F}_{n}(x)\right\}} V_{d}\left(\left[F_{1}, x\right]\right)^{2} \prod_{j=1}^{n} \mathrm{~d} \phi_{d}\left(x_{j}\right) \mathrm{d} \phi_{d}(x) \\
& \leq n^{d} \int_{\mathbb{R}^{d}} \cdots \int_{\mathbb{R}^{d}} \Phi\left(h_{1}\right)^{n-d} \mathbf{1}_{\left\{1 \leq h_{1}\right\}} \\
& \quad \times \int_{\mathbb{R}^{d}} \mathbf{1}_{\left\{x \in H_{+}\left(F_{1}\right)\right\}}\left(\left\langle x, u_{1}\right\rangle-h_{1}\right)^{2} \mathrm{~d} \phi_{d}(x) \lambda_{H_{1}}\left(F_{1}\right)^{2} \prod_{j=1}^{d} \mathrm{~d} \phi_{d}\left(x_{j}\right) \\
& =n^{d} \int_{\mathbb{R}^{d}} \cdots \int_{\mathbb{R}^{d}} \Phi\left(h_{1}\right)^{n-d} \mathbf{1}_{\left\{1 \leq h_{1}\right\}} \int_{h_{1}}^{\infty}\left(h-h_{1}\right)^{2} \phi(h) \mathrm{d} h \lambda_{H_{1}}\left(F_{1}\right)^{2} \prod_{j=1}^{d} \mathrm{~d} \phi_{d}\left(x_{j}\right) \\
& \leq c_{3} n^{d} \int_{\mathbb{R}^{d}} \cdots \int_{\mathbb{R}^{d}} \Phi\left(h_{1}\right)^{n-d} \mathbf{1}_{\left\{1 \leq h_{1}\right\}} h_{1}^{-3} \phi\left(h_{1}\right) \lambda_{H_{1}}\left(F_{1}\right)^{2} \prod_{j=1}^{d} \mathrm{~d} \phi_{d}\left(x_{j}\right) \\
& \leq c_{4} n^{d} \int_{1}^{\infty} \Phi\left(h_{1}\right)^{n-d} h_{1}^{-3} \phi\left(h_{1}\right)^{d+1} \mathrm{~d} h_{1} \\
& \leq c_{5} n^{-1}(\ln n)^{(d-3) / 2},
\end{aligned}
$$

where the affine Blaschke-Petkantschin formula and Lemma 4.2 have been applied in the fourth step.

If $d=1$ then only the case $k=1$ can occur, and the proof is finished at this point. Subsequently, we consider the case $d \geq 2$. In order to estimate the summands corresponding to $k$ with $0 \leq k \leq d-1$, we need some preparations. Again, denote by $\varphi$ the angle between $u_{1}$ and $u_{2}$, by $\bar{h}_{12}$ the distance from $H_{+}\left(F_{1}\right) \cap H_{+}\left(F_{2}^{(k)}\right)$ to the origin, and by $u_{12}$ the unit vector pointing from the origin to the nearest point of $H_{+}\left(F_{1}\right) \cap H_{+}\left(F_{2}^{(k)}\right)$. Then $H_{+}\left(F_{1}\right) \cap H_{+}\left(F_{2}^{(k)}\right)$ is contained in $\left\{y \in \mathbb{R}^{d}:\left\langle y, u_{12}\right\rangle \geq h_{12}\right\}$. We use the parametrization $x=h u_{12}+z$ with $z \in u_{12}^{\perp}$, where $h \geq h_{12}$, and have

$$
\left\langle x, u_{i}\right\rangle-h_{i}=h\left\langle u_{12}, u_{i}\right\rangle+\left\langle z, u_{i}\right\rangle-h_{i} \leq h-h_{12}+\|z\| \sin \varphi+h_{12}\left\langle u_{12}, u_{i}\right\rangle-h_{i}
$$

for $\varphi \leq \frac{1}{2} \pi$, since the angle between $u_{i}$ and $u_{12}$ is bounded by the angle between $u_{1}$ and $u_{2}$. If $\varphi^{*} \leq \varphi \leq \frac{1}{2} \pi$ then $h_{12}=h_{12}^{(d-2)}$ and $h_{12} u_{12} \in H\left(F_{1}\right) \cap H\left(F_{2}^{(k)}\right)$; hence, $h_{12}\left\langle u_{12}, u_{i}\right\rangle=h_{i}$ for $i=1,2$. If $0<\varphi<\varphi^{*}$ then $h_{12} u_{12} \in H\left(F_{2}^{(k)}\right), h_{12}=h_{2}, u_{12}=u_{2}$, and $h_{12}\left\langle u_{12}, u_{1}\right\rangle-h_{1} \leq$ $h_{2}-h_{1}$. In either case, we then have

$$
\begin{aligned}
\int_{\mathbb{R}^{d}} & \mathbf{1}_{\left\{x \in H_{+}\left(F_{1}\right) \cap H_{+}\left(F_{2}^{(k)}\right)\right\}}\left(\left\langle x, u_{1}\right\rangle-h_{1}\right)\left(\left\langle x, u_{2}\right\rangle-h_{2}\right) \mathrm{d} \phi_{d}(x) \\
& \leq \int_{h_{12}}^{\infty} \int_{u_{12}^{\perp}}\left(h-h_{12}+\|z\| \sin \varphi+h_{2}-h_{1}\right)\left(h-h_{12}+\|z\| \sin \varphi\right) \mathrm{d} \phi_{d-1}(z) \mathrm{d} \phi(h) \\
& \leq c_{6}\left(h_{12}^{-3}+h_{12}^{-2}\left(\sin \varphi+\left(h_{2}-h_{1}\right)\right)+h_{12}^{-1}\left(\sin ^{2} \varphi+\left(h_{2}-h_{1}\right) \sin \varphi\right)\right) \phi\left(h_{12}\right) \\
& =: S_{1}\left(h_{1}, h_{2}, \varphi\right)
\end{aligned}
$$

for $\varphi \leq \frac{1}{2} \pi$, where we have used Lemma 4.1. For $\varphi \geq \frac{1}{2} \pi$, (6.9) implies that

$$
h_{12}^{(d-2)}=h_{12} \geq 2^{1 / 2} h_{1} .
$$


Thus, for $\varphi \geq \frac{1}{2} \pi$ we obtain

$$
\begin{aligned}
\int_{\mathbb{R}^{d}} & \mathbf{1}_{\left\{x \in H_{+}\left(F_{1}\right) \cap H_{+}\left(F_{2}^{(k)}\right)\right\}}\left(\left\langle x, u_{1}\right\rangle-h_{1}\right)\left(\left\langle x, u_{2}\right\rangle-h_{2}\right) \mathrm{d} \phi_{d}(x) \\
& \leq \int_{h_{12}}^{\infty}\left(h-h_{1}\right)^{2} \phi(h) \mathrm{d} h \leq 3 \phi\left(h_{12}\right)+\frac{\left(h_{12}-h_{1}\right)^{2}}{h_{12}} \phi\left(h_{12}\right) \\
& \leq c_{7} \phi\left(\eta h_{1}\right) \\
& =: S_{2}\left(h_{1}, h_{2}, \varphi\right),
\end{aligned}
$$

where $\eta:=\frac{1}{2}\left(1+2^{1 / 2}\right)$. In addition, we define

$$
\begin{array}{ll}
S_{1}\left(h_{1}, h_{2}, \varphi\right)=0 & \text { for } \varphi>\frac{1}{2} \pi, \\
S_{2}\left(h_{1}, h_{2}, \varphi\right)=0 & \text { for } \varphi<\frac{1}{2} \pi,
\end{array}
$$

and then let

$$
S\left(h_{1}, h_{2}, \varphi\right):=S_{1}\left(h_{1}, h_{2}, \varphi\right)+S_{2}\left(h_{1}, h_{2}, \varphi\right) .
$$

The probability that the random points $X_{2 d-k+1}, \ldots, X_{n}$ are contained in $H_{0}\left(F_{1}\right) \cap H_{0}\left(F_{2}^{(k)}\right)$ can be bounded from above by $\Phi\left(h_{1}\right)^{n-2 d+k}$. Hence, we obtain

$$
\begin{aligned}
& \mathrm{E}\left(V_{d}\left(P_{n+1}\right)-V_{d}\left(P_{n}\right)\right)^{2} \\
& \leq c_{8} \sum_{k=0}^{d-1} n^{2 d-k} \int_{\mathbb{R}^{d}} \cdots \int_{\mathbb{R}^{d}} \mathbf{1}_{\left\{1 \leq h_{1} \leq h_{2}\right\}} \Phi\left(h_{1}\right)^{n-2 d+k} S\left(h_{1}, h_{2}, \varphi\right) \\
& \quad \times \lambda_{H_{1}}\left(F_{1}\right) \lambda_{H_{2}}\left(F_{2}^{(k)}\right) \prod_{j=1}^{2 d-k} \mathrm{~d} \phi_{d}\left(x_{j}\right)+O\left(n^{-1}(\ln n)^{(d-3) / 2}\right) .
\end{aligned}
$$

For $k \in\{0, \ldots, d-1\}$, we apply the Blaschke-Petkantschin formula (3.1) to the integral

$$
\tau_{k}=\int_{\mathbb{R}^{d}} \cdots \int_{\mathbb{R}^{d}} \mathbf{1}_{\left\{1 \leq h_{1} \leq h_{2}\right\}} \Phi\left(h_{1}\right)^{n-2 d+k} S\left(h_{1}, h_{2}, \varphi\right) \lambda_{H_{1}}\left(F_{1}\right) \lambda_{H_{2}}\left(F_{2}^{(k)}\right) \prod_{j=1}^{2 d-k} \mathrm{~d} \phi_{d}\left(x_{j}\right)
$$

to obtain

$$
\begin{aligned}
I_{k}=\Gamma(d)^{2} \int_{\mathbb{S}^{d-1}} & \int_{1}^{\infty} \int_{\mathbb{S}^{d-1}} \int_{h_{1}}^{\infty} \Phi\left(h_{1}\right)^{n-2 d+k} S\left(h_{1}, h_{2}, \varphi\right)(\sin \varphi)^{-k} \\
& \times \mathscr{g}_{k}\left(u_{1}, h_{1}, u_{2}, h_{2}\right) \mathrm{d} h_{2} \mathrm{~d} \sigma\left(u_{2}\right) \mathrm{d} h_{1} \mathrm{~d} \sigma\left(u_{1}\right),
\end{aligned}
$$

with

$$
\begin{aligned}
g_{k}\left(u_{1}, h_{1}, u_{2}, h_{2}\right) & \\
= & \int_{H_{1}} \cdots \int_{H_{1}} \int_{H_{1} \cap H_{2}} \cdots \int_{H_{1} \cap H_{2}} \int_{H_{2}} \ldots \int_{H_{2}} \lambda_{H_{1}}\left(F_{1}\right)^{2} \lambda_{H_{2}}\left(F_{2}^{(k)}\right)^{2} \prod_{j=1}^{2 d-k} \phi_{d}\left(x_{j}\right) \\
& \times \prod_{j=d+1}^{2 d-k} \mathrm{~d} \lambda_{H_{2}}\left(x_{j}\right) \prod_{j=d-k+1}^{d} \mathrm{~d} \lambda_{H_{1} \cap H_{2}}\left(x_{j}\right) \prod_{j=1}^{d-k} \mathrm{~d} \lambda_{H_{1}}\left(x_{j}\right) .
\end{aligned}
$$


The two cases $k=0$ and $k \in\{1, \ldots, d-1\}$ will again be treated separately. We decompose $I_{k}$ in the form $I_{k}=I_{k}^{1}+I_{k}^{2}$, corresponding to the decomposition $S=S_{1}+S_{2}$.

We start with the case $k=0$, and obtain

$$
\begin{aligned}
\tau_{0}^{1}=c_{9} \int_{\mathbb{S}^{d-1}} & \int_{1}^{\infty} \int_{\mathbb{S}^{d-1}} \int_{h_{1}}^{\infty} \Phi\left(h_{1}\right)^{n-2 d} S_{1}\left(h_{1}, h_{2}, \varphi\right) \\
& \times \int_{H_{1}} \ldots \int_{H_{1}} \lambda_{H_{1}}\left(F_{1}\right)^{2} \prod_{j=1}^{d} \phi_{d}\left(x_{j}\right) \prod_{j=1}^{d} \mathrm{~d} \lambda_{H_{1}}\left(x_{j}\right) \\
& \times \int_{H_{2}} \cdots \int_{H_{2}} \lambda_{H_{2}}\left(F_{2}^{(0)}\right)^{2} \prod_{j=d+1}^{2 d} \phi_{d}\left(x_{j}\right) \prod_{j=d+1}^{2 d} \mathrm{~d} \lambda_{H_{2}}\left(x_{j}\right) \mathrm{d} h_{2} \mathrm{~d} \sigma\left(u_{2}\right) \mathrm{d} h_{1} \mathrm{~d} \sigma\left(u_{1}\right) \\
\leq & c_{10} \int_{\mathbb{S}^{d-1}} \int_{1}^{\infty} \int_{\mathbb{S}^{d-1}} \int_{h_{1}}^{\infty} \Phi\left(h_{1}\right)^{n-2 d} S_{1}\left(h_{1}, h_{2}, \varphi\right) \phi\left(h_{1}\right)^{d} \phi\left(h_{2}\right)^{d} \mathrm{~d} h_{2} \mathrm{~d} \sigma\left(u_{2}\right) \mathrm{d} h_{1} \mathrm{~d} \sigma\left(u_{1}\right) \\
\leq & c_{11} \int_{1}^{\infty} \Phi\left(h_{1}\right)^{n-2 d} \phi\left(h_{1}\right)^{d} \int_{0}^{\pi / 2} \int_{h_{1}}^{\infty} S_{1}\left(h_{1}, h_{2}, \varphi\right)(\sin \varphi)^{d-2} \phi\left(h_{2}\right)^{d} \mathrm{~d} h_{2} \mathrm{~d} \varphi \mathrm{d} h_{1} .
\end{aligned}
$$

Now we must consider the five different summands into which $S_{1}\left(h_{1}, h_{2}, \varphi\right)$ naturally decomposes, and estimate the corresponding integrals. Using the fact that $h_{12} \geq h_{1}$ and repeatedly applying Lemma 4.3, we thus obtain

$$
\int_{h_{1}}^{\infty} \int_{0}^{\pi / 2} S_{1}\left(h_{1}, h_{2}, \varphi\right)(\sin \varphi)^{d-2} \phi\left(h_{2}\right)^{d} \mathrm{~d} h_{2} \mathrm{~d} \varphi \leq c_{12} h_{1}^{-(d+3)} \phi\left(h_{1}\right)^{d+1}
$$

and, so, arrive at

$$
I_{0}^{1} \leq c_{13} \int_{1}^{\infty} \Phi\left(h_{1}\right)^{n-2 d} h_{1}^{-(d+3)} \phi\left(h_{1}\right)^{2 d+1} \mathrm{~d} h_{1} \leq c_{14} n^{-(2 d+1)}(\ln n)^{(d-3) / 2} .
$$

Moreover,

$$
\begin{aligned}
\tau_{0}^{2} & \leq c_{15} \int_{\mathbb{S}^{d-1}} \int_{1}^{\infty} \int_{\mathbb{S}^{d-1}} \int_{h_{1}}^{\infty} \Phi\left(h_{1}\right)^{n-2 d} S_{2}\left(h_{1}, h_{2}, \varphi\right) \phi\left(h_{1}\right)^{d} \phi\left(h_{2}\right)^{d} \mathrm{~d} h_{2} \mathrm{~d} \sigma\left(u_{2}\right) \mathrm{d} h_{1} \mathrm{~d} \sigma\left(u_{1}\right) \\
& \leq c_{16} \int_{1}^{\infty} \Phi\left(h_{1}\right)^{n-2 d} \phi\left(h_{1}\right)^{d} \int_{\pi / 2}^{\pi} \int_{h_{1}}^{\infty} \phi\left(\eta h_{1}\right)(\sin \varphi)^{d-2} \phi\left(h_{2}\right)^{d} \mathrm{~d} h_{2} \mathrm{~d} \varphi \mathrm{d} h_{1} \\
& \leq c_{17} \int_{1}^{\infty} \Phi\left(h_{1}\right)^{n-2 d} h_{1}^{-1} \phi\left(h_{1}\right)^{2 d+\eta^{2}} \mathrm{~d} h_{1} \\
& \leq c_{18} n^{-\left(2 d+\eta^{2}\right)}(\ln n)^{\left(2 d+\eta^{2}-1-1\right) / 2} \\
& \leq c_{19} n^{-(2 d+1)}(\ln n)^{(d-3) / 2},
\end{aligned}
$$

since $1<\eta^{2}<2$. Thus, we have

$$
I_{0} \leq c_{20} n^{-(2 d+1)}(\ln n)^{(d-3) / 2} .
$$

We next consider the cases $k \in\{1, \ldots, d-1\}$. First, we obtain

$$
g_{k}\left(u_{1}, h_{1}, u_{2}, h_{2}\right) \leq c_{21} \phi\left(h_{1}\right)^{d-k+1 / 2} \phi\left(h_{2}\right)^{d-k} \phi\left(h_{12}^{(d-2)}\right)^{k-1 / 2}
$$


by arguing in a similar way as we did in proving (6.7). We therefore have to bound

$$
\begin{aligned}
& \int_{1}^{\infty} \int_{\mathbb{S}^{d-1}} \int_{h_{1}}^{\infty} \Phi\left(h_{1}\right)^{n-2 d+k} \\
& \quad \times S\left(h_{1}, h_{2}, \varphi\right)(\sin \varphi)^{-k} \phi\left(h_{1}\right)^{d-k+1 / 2} \phi\left(h_{2}\right)^{d-k} \phi\left(h_{12}^{(d-2)}\right)^{k-1 / 2} \mathrm{~d} h_{2} \mathrm{~d} \sigma\left(u_{2}\right) \mathrm{d} h_{1}
\end{aligned}
$$

from above, where $\varphi$ is the angle between $e_{1}$ and $u_{2}$. Parametrizing the unit sphere as before, we see that this integral can be bounded from above by

$$
\begin{aligned}
& \int_{1}^{\infty} \int_{h_{1}}^{\infty} \int_{0}^{\infty} \Phi\left(h_{1}\right)^{n-2 d+k} \phi\left(h_{1}\right)^{d-k+1 / 2} \\
& \quad \times S\left(h_{1}, h_{2}, \varphi\right) \phi\left(h_{2}\right)^{d-k} \phi\left(h_{12}^{(d-2)}\right)^{k-1 / 2}(\sin \varphi)^{d-k-2} \mathrm{~d} \varphi \mathrm{d} h_{2} \mathrm{~d} h_{1},
\end{aligned}
$$

up to a constant multiplier. Using (6.9), Lemma 4.4, and the facts that $h_{12} \geq h_{1}, h_{2} \geq h_{1}$, and $k-\frac{1}{2} \geq \frac{1}{4}$, this multiple integral can be estimated from above by

$$
\begin{aligned}
\int_{1}^{\infty} & \Phi\left(h_{1}\right)^{n-2 d+k} \phi\left(h_{1}\right)^{d-k+1 / 2} \phi\left(h_{1}\right)^{k-1 / 2} \phi\left(h_{1}\right) \\
& \times \int_{h_{1}}^{\infty} \int_{0}^{\infty} \phi\left(h_{2}\right)^{d-k} S\left(h_{1}, h_{2}, \varphi\right) \phi\left(-h_{12}\right) \phi\left(\frac{h_{1}-h_{2} \cos \varphi}{2 \sin \varphi}\right)(\sin \varphi)^{d-k-2} \mathrm{~d} \varphi \mathrm{d} h_{2} \mathrm{~d} h_{1} \\
\leq & c_{22} \int_{1}^{\infty} \Phi\left(h_{1}\right)^{n-2 d+k} \phi\left(h_{1}\right)^{d+1} h_{1}^{-(d-k+3)} \phi\left(h_{1}\right)^{d-k} \mathrm{~d} h_{1} \\
\leq & c_{23} n^{-2 d+k-1}(\ln n)^{(d-3) / 2}
\end{aligned}
$$

Thus, combining (7.1), (7.2), (7.3), and (7.4), we finally obtain

$$
\mathrm{E}\left(V_{d}\left(P_{n+1}\right)-V_{d}\left(P_{n}\right)\right)^{2} \leq c_{24} n^{-1}(\ln n)^{(d-3) / 2},
$$

which completes the proof.

\section{References}

[1] Affentranger, F. (1991). The convex hull of random points with spherically symmetric distributions. Rend. Sem. Mat. Univ. Politec. Torino 49, 359-383.

[2] Affentranger, F. and Schneider, R. (1992). Random projections of regular simplices. Discrete Comput. Geom. 7, 219-226.

[3] Baryshnikov, Y. M. and Vitale, R. A. (1994). Regular simplices and Gaussian samples. Discrete Comput. Geom. 11, 141-147.

[4] Efron, B. and Stein, C. (1981). The jackknife estimate of variance. Ann. Statist. 9, 586-596.

[5] Geffroy, J. (1961). Localisation asymptotique du polyèdre d'appui d'un échantillon Laplacien à $k$ dimensions. Publ. Inst. Statist. Univ. Paris 10, 213-228.

[6] Henze, N. And KLeIn, T. (1996). The limit distribution of the largest interpoint distance from a symmetric Kotz sample. J. Multivariate Anal. 57, 228-239.

[7] Hueter, I. (1994). The convex hull of a normal sample. Adv. Appl. Prob. 26, 855-875.

[8] Hueter, I. (1999). Limit theorems for the convex hull of random points in higher dimensions. Trans. Amer. Math. Soc. 351, 4337-4363.

[9] Hug, D., Munsonius, G. O. And Reitzner, M. (2004). Asymptotic mean values of Gaussian polytopes. Beiträge Algebra Geom. 45, 531-548.

[10] HüsLER, J. (1997). Range of bivariate normal random vectors. Rend. Circ. Mat. Palermo (2) Suppl. 50, $229-234$. 
[11] Jensen, E. B. V. (1998). Local Stereology (Adv. Ser. Statist. Sci. Appl. Prob. 5). World Scientific, River Edge, NJ.

[12] Jensen, E. B. V. AND KIÊU, K. (1992). A new integral geometric formula of the Blaschke-Petkantschin type. Math. Nachr. 156, 57-74.

[13] Kendall, M. G. and Stuart, A. (1965). The Advanced Theory of Statistics: Distribution Theory, Vol. 1, 3rd edn. Griffin, London.

[14] Mardia, K. V. (1965). Tippet's formulas and other results on sample range and extremes. Ann. Inst. Statist. Math. 17, 85-91.

[15] Massé, B. (2000). On the LLN for the number of vertices of a random convex hull. Adv. Appl. Prob. 32, 675-681.

[16] Matthews, P. C. And Rukhin, A. L. (1993). Aysmptotic distribution of the normal sample range. Ann. Appl. Prob. 3, 454-466.

[17] Miles, R. E. (1971). Isotropic random simplices. Adv. Appl. Prob. 3, 353-382.

[18] Patel, J. K. And Read, C. B. (1996). Handbook of the Normal Distribution (Statist. Textbook Monogr. 40). Marcel Dekker, New York.

[19] Raynaud, H. (1970). Sur l'enveloppe convexe des nuages de points aléatoires dans $\mathbb{R}^{n}$. I. J. Appl. Prob. 7, $35-48$.

[20] Reitzner, M. (2003). Random polytopes and the Efron-Stein jackknife inequality. Ann. Prob. 31, $2136-2166$.

[21] Reitzner, M. (2005). The combinatorial structure of random polytopes. Adv. Math. 191, 178-208.

[22] RÉNYI, A. AND Sulanke, R. (1963). Über die konvexe Hülle von $n$ zufällig gewählten Punkten. Z. Wahrscheinlichkeitsth. 2, 75-84.

[23] Santaló, L. A. (1976). Integral Geometry and Geometric Probability. Addison-Wesley, Reading, MA.

[24] SchneIder, R. (1993). Convex Bodies: the Brunn-Minkowski Theory (Encyclopedia Math. Appl. 44). Cambridge University Press.

[25] SchneIder, R. (1997). Discrete aspects of stochastic geometry. In Handbook of Discrete and Computational Geometry, eds J. E. Goodman and J. O’Rourke, CRC, Boca Raton, FL, pp. 255-278.

[26] SchneIDER, R. AND WeIL, W. (1992). Integralgeometrie. Teubner, Stuttgart.

[27] Vershik, A. M. AND Sporyshev, P. V. (1992). Asymptotic behavior of the number of faces of random polyhedra and the neighborliness problem. Select. Math. Soviet. 11, 181-201.

[28] Wendel, J. G. (1962). A problem in geometric probability. Math. Scand. 11, 109-111. 